\title{
Cutting resilient networks - complete binary trees
}

\author{
Xing Shi Cai Cecilia Holmgren* \\ The Department Mathematics \\ Uppsala University \\ Uppsala, Sweden \\ \{xingshi.cai, cecilia.holmgren\}@math.uu.se
}

Submitted: Nov 29, 2018; Accepted: Oct 28, 2019; Published: Dec 6, 2019

(C) The authors. Released under the CC BY-ND license (International 4.0).

\begin{abstract}
In our previous work $[2,3]$, we introduced the random $k$-cut number for rooted graphs. In this paper, we show that the distribution of the $k$-cut number in complete binary trees of size $n$, after rescaling, is asymptotically a periodic function of $\lg n-$ $\lg \lg n$. Thus there are different limit distributions for different subsequences, where these limits are similar to weakly 1 -stable distributions. This generalizes the result for the case $k=1$, i.e., the traditional cutting model, by Janson [12].

Keywords: complete binary tree, infinitely divisible distributions, stable distributions, cuttings of trees
\end{abstract}

Mathematics Subject Classifications: 60C05, 60F05, 05C05

\section{Introduction}

\subsection{The model and the motivation}

In our previous work $[2,3]$, we introduced the $k$-cut number for rooted graphs. Let $k$ be an integer. Let $G_{n}$ be a connected graph of $n$ nodes with exactly one node labelled as the root. We remove nodes from the graph using this random procedure (note that in our model nodes are only removed after having been cut $k$ times):

1. Initially set every node's cut-counter to zero, i.e., no node has ever been cut.

2. Choose one node uniformly at random from the component containing the root and increase its cut-counter by one, i.e., we cut the selected node once.

*This work was partially supported by two grants from the Knut and Alice Wallenberg Foundation, a grant from the Swedish Research Council, and the Swedish Foundations' starting grant from the Ragnar Söderberg Foundation. 
3. If this node's cut-counter hits $k$, i.e., it has been cut $k$ times, then remove it from the graph.

4. If the root has been removed, then stop. Otherwise, go to step 2.

We call the (random) total number of cuts needed for this procedure to end the $k$-cut number and denote it by $\mathcal{K}\left(G_{n}\right)$. The traditional cutting model corresponds to the case that $k=1$.

We can also cut and remove edges instead of nodes using the same process with the modification that we stop when the root has been isolated. We denote the total number of cuts needed for this edge removing process to end by $\mathcal{K}^{e}\left(G_{n}\right)$.

The $k$-cut number can be seen as a measure of the difficulty for the destruction of a resilient network. For example, in a botnet, a bot-master controls a large number of compromised computer (bots) for various cybercrimes. To counter attack a botnet means to reduce the number of bots reachable from the bot-master by fixing compromised computers [5]. We can view a botnet as a graph and fixing a computer as removing a node from the graph. If we assume that each compromised computer takes $k$-attempts to clean, and each attempt aims at a computer chosen uniformly at random, then the $k$-cut number is precisely the number of attempts of cleaning up needed to completely destroy a botnet.

The case $k=1$, i.e., the traditional cutting model has been well-studied. It was first introduced by Meir and Moon [17] for uniform random Cayley trees. Janson [12,13] studied one-cuts in binary trees and conditioned Galton-Watson trees. Addario-Berry, Broutin and Holmgren [1] simplified the proof for the limit distribution of one-cuts in conditioned Galton-Watson trees. The cutting model has also been studied in random recursive trees, see Meir and Moon [16], Iksanov and Möhle [11], and Drmota, Iksanov, Moehle and Roesler [7]. For binary search trees and split trees, see Holmgren [9, 10].

In our previous work [3], we mainly analyzed $\mathcal{K}\left(P_{n}\right)$, the $k$-cut number for a path of length $n$, which generalizes the record number in a uniform random permutation. In this paper, we continue our investigation in complete binary trees, i.e., binary trees in which each level is full except possibly for the last level, and the nodes at the last level occupy the leftmost positions. If the last level is also full, then we call the tree a full binary tree.

\subsection{An equivalent model}

Let $T_{n}^{\text {bin }}$ be a complete binary tree of size $n$. Let $\mathcal{X}_{n} \stackrel{\text { def }}{=} \mathcal{K}\left(T_{n}^{\text {bin }}\right)$ and $\mathcal{X}_{n}^{e} \stackrel{\text { def }}{=} \mathcal{K}^{e}\left(T_{n}^{\text {bin }}\right)$ with the root of the tree as the root of the graph. There is an equivalent way to define $\mathcal{X}_{n}$. Let $\left(E_{r, v}, r \geqslant 1, v \in T_{n}^{\text {bin }}\right)$ be i.i.d. exponential random variables with mean 1 . Let $T_{r, v} \stackrel{\text { def }}{=} \sum_{j=1}^{r} E_{j, v}$. Imagine each node in $T_{n}^{\text {bin }}$ has an alarm clock and node $v$ 's clock fires at times $\left(T_{r, v}, r \geqslant 1\right)$. If we cut a node when its alarm clock fires, then due to the memoryless property of exponential random variables, we are actually choosing a node uniformly at random to cut.

However, this also means that we are cutting nodes that have already been removed from the tree. Thus for a cut on node $v$ at time $T_{r, v}$ (for some $r \leqslant k$ ) to be counted in 
$\mathcal{X}_{n}$, none of its ancestors can have already been cut $k$ times, i.e.,

$$
T_{r, v}<\min _{u: u \prec v} T_{k, u}
$$

where $u \prec v$ denotes that $u$ is an ancestor of $v$. When the event in (1.1) happens, we say that $T_{r, v}$ (or simply $v$ ) is an $r$-record and let $I_{r, v}$ be the indicator random variable for this event. Let $\mathcal{X}_{n}^{r}$ be the total number of $r$-records, i.e., $\mathcal{X}_{n}^{r} \stackrel{\text { def }}{=} \sum_{v} I_{r, v}$. Then obviously $\mathcal{X}_{n} \stackrel{\mathcal{L}}{=} \sum_{r=1}^{k} \mathcal{X}_{n}^{r}$. We use this equivalence for the rest of the paper.

By assigning alarm clocks to edges instead of nodes, we can define the edge version of $r$-records $\mathcal{X}_{n}^{e, r}$ and have $\mathcal{X}_{n}^{e} \stackrel{\mathcal{L}}{=} \sum_{r=1}^{k} \mathcal{X}_{n}^{e, r}$.

\subsection{The main results}

To introduce the main results, we need some notations. Let $\{x\}$ denote the fractional part of $x$, i.e., $\{x\} \stackrel{\text { def }}{=} x-\lfloor x\rfloor$. Let $\Gamma(a)$ be the Gamma function $[6,5.2 .1]$. Let $\Gamma(a, x)$ be the upper incomplete Gamma function [6, 8.2.2]. Let $Q(a, x) \stackrel{\text { def }}{=} \Gamma(a, x) / \Gamma(a)$. Let $Q^{-1}(a, x)$ be the inverse of $Q(a, x)$ with respect to $x$. Let $\lg (x) \stackrel{\text { def }}{=} \log _{2}(x)$.

Theorem 1.1. Assume that $\{\lg n-\lg \lg n\} \rightarrow \gamma \in[0,1]$ as $n \rightarrow \infty$. Then

$$
\frac{\lg (n)^{\frac{r}{k}+1}}{C_{2}(r) n} \mathcal{X}_{n}^{r}-\mu_{r, n} \stackrel{d}{\rightarrow} 1-C_{3}(r) W_{r, k, \gamma}
$$

where

$$
\mu_{r, n}=\frac{k}{r} \lg (n)+\sum_{i=1}^{k} C_{1}(r, i) \lg (n)^{1-\frac{i}{k}}+\lg (\lg (n)),
$$

$C_{1}(\cdot, \cdot), C_{2}(\cdot, \cdot)$, and $C_{3}(\cdot)$ are constants defined in Proposition 4.1, and $W_{r, k, \gamma}$ has an infinitely divisible distribution with the characteristic function

$$
\mathbb{E}\left[\exp \left(\mathrm{i} t W_{r, k, \gamma}\right)\right]=\exp \left(\mathrm{i} f_{r, k, \gamma} t+\int_{0}^{\infty}\left(\mathrm{e}^{\mathrm{i} t x}-1-\mathrm{i} t x \cdot \mathbb{1}[x<1]\right) \mathrm{d} \nu_{r, k, \gamma}(x)\right),
$$

where $f_{r, k, \gamma}$ is a constant defined later in (5.39) and the Lévy measure $\nu_{r, k, \gamma}$ has support on $(0, \infty)$ with density

$$
\begin{gathered}
\frac{\mathrm{d} \nu_{r, k, \gamma}}{\mathrm{d} x}=\frac{\Gamma\left(\frac{r}{k}\right)^{2}}{x^{2}} \sum_{s \geqslant 1} 4^{\left\{\gamma+\lg \left(x / \Gamma\left(\frac{r}{k}\right)\right)\right\}-s} \exp \left(Q^{-1}\left(\frac{r}{k}, 2^{\left\{\gamma+\lg \left(x / \Gamma\left(\frac{r}{k}\right)\right)\right\}-s}\right)\right) \\
Q^{-1}\left(\frac{r}{k}, 2^{\left\{\gamma+\lg \left(x / \Gamma\left(\frac{r}{k}\right)\right)\right\}-s}\right)^{1-\frac{r}{k}} .
\end{gathered}
$$

Theorem 1.2. Assume the same conditions as in Theorem 1.1. Then

$$
\frac{\lg (n)^{\frac{1}{k}+1}}{C_{2}(1) n}\left(\mathcal{X}_{n}-\sum_{r=1}^{k} \frac{C_{2}(r) n}{\lg (n)^{\frac{r}{k}+1}} \mu_{r, n}\right) \stackrel{d}{\rightarrow} 1-C_{3}(1) W_{1, k, \gamma} .
$$

The same holds true for $\mathcal{X}_{n}^{e}$. 
Remark 1.1. Let $\widetilde{X}_{n}$ denote the left-hand-side of (1.6). Another way of formulating Theorem 1.2 is by saying that the distance, e.g., in the Lévy metric, between the distribution of $\widetilde{X}_{n}$ and the distribution of $1-C_{3}(1) W_{1, k,\{\lg n-\lg \lg n\}}$ tends to zero as $n \rightarrow \infty$.

Remark 1.2. We do not have a closed form for $C_{1}(\cdot, \cdot)$. But for specific $k$ they are easy to compute with computer algebra systems. When $k=r=1$, i.e., when $\mathcal{X}_{n}^{1}=\mathcal{X}_{n},(1.6)$ reduces to

$$
\mathcal{X}_{n} \frac{\lg (n)^{2}}{n}-\lg (n)-\lg (\lg (n)) \stackrel{d}{\rightarrow}-W_{1,1, \gamma}
$$

and since $Q^{-1}(1, x)=\log (1 / x),(1.5)$ reduces to

$$
\frac{\mathrm{d} \nu_{1,1, \gamma}}{\mathrm{d} x}=\frac{1}{x^{2}} 2^{\{\lg x+\gamma\}} .
$$

In other words, we recover the result for the traditional cutting model in complete binary trees by Janson [12, Theorem 1.1]. When $k=2$, (1.6) reduces to

$$
\sqrt{\frac{8}{\pi}} \frac{\lg (n)^{\frac{3}{2}}}{n} \mathcal{X}_{n}-2 \lg (n)-\frac{1}{3} \sqrt{\frac{2}{\pi}} \lg (n)^{\frac{1}{2}}-\lg (\lg (n))-\frac{11}{3} \stackrel{d}{\rightarrow}-\frac{2 W_{1,2, \gamma}}{\sqrt{\pi}} .
$$

Remark 1.3. In Remark 1.5 of [12], Janson mentioned that when $k=r=1$, if $W_{1,1, \gamma}^{\prime}$ and $W_{1,1, \gamma}^{\prime \prime}$ are independent copies of $W_{1,1, \gamma}$, then $W_{1,1, \gamma}^{\prime}+W_{1,1, \gamma}^{\prime \prime} \stackrel{\mathcal{L}}{=} 2 W_{1,1, \gamma}+2$, but the corresponding statement for three copies of $W_{1,1, \gamma}$ is false. In other words, $W_{1,1, \gamma}$ is roughly similar to a 1-stable distribution. This extends to general $k$ in the sense that

$$
W_{r, k, \gamma}^{\prime}+W_{r, k, \gamma}^{\prime \prime} \stackrel{\mathcal{L}}{=} 2 W_{r, k, \gamma}+2 \int_{1}^{2} x \mathrm{~d} \nu_{r, k, \gamma}(x)
$$

with $\int_{1}^{2} x \mathrm{~d} \nu_{1,1, \gamma}(x)=1$. This follows by computing the characteristic functions of both sides using (1.4) and by noticing that

$$
\left.\frac{\mathrm{d} \nu_{r, k, \gamma}}{\mathrm{d} x}\right|_{x=u}=\left.\frac{1}{4} \frac{\mathrm{d} \nu_{r, k, \gamma}}{\mathrm{d} x}\right|_{x=\frac{u}{2}} .
$$

In the rest of the paper, we will first compute the expected number and variance of $r$-records conditioning on $T_{k, o}=y$, where $o$ denotes the root. Then we show that the fluctuation of the total number of $r$-records from its mean is more or less the same as the sum of such fluctuations in each subtree rooted at height $L \stackrel{\text { def }}{=}\left\lfloor\left(2-\frac{1}{2 k}\right) \lg \lg n\right\rfloor$, conditioning on what happens below height $L$. This sum can be further approximated by a sum of independent random variables. Finally, we apply a classic theorem regarding the convergence to infinitely divisible distributions by Kallenberg [15, Theorem 15.23] to prove Theorem 1.1 and Theorem 1.2.

The proof follows a similar path as Janson [12] did for the case $k=1$. However, the analysis for $k \geqslant 2$ is significantly more complicated.

Holmgren $[9,10]$ showed that when $k=1, \mathcal{X}_{n}$ has similar behaviour in binary search trees and split trees as in complete binary trees. We are currently trying to prove this for $k \geqslant 2$. 


\section{Some more notations}

We collect some of the notations which are used frequently in this paper.

Let $\Gamma(a)$ be the Gamma function $[6,5.2 .1]$, i.e.,

$$
\Gamma(a)=\int_{0}^{\infty} \mathrm{e}^{-t} t^{a-1} \mathrm{~d} t, \quad \operatorname{Re}(a)>0 .
$$

Note that $\Gamma(k)=k$ ! for $k \in \mathbb{N}$. Let $\Gamma(a, x)$ and $\gamma(a, x)$ be the upper and lower incomplete Gamma functions respectively [6, 8.2], i.e.,

$$
\Gamma(a, z)=\int_{z}^{\infty} \mathrm{e}^{-t} t^{a-1} \mathrm{~d} t, \quad \gamma(a, z)=\int_{0}^{z} \mathrm{e}^{-t} t^{a-1} \mathrm{~d} t, \quad \operatorname{Re}(a)>0 .
$$

Thus $\gamma(a, x) \stackrel{\text { def }}{=} \Gamma(a)-\Gamma(a, x)$. Let $\Gamma\left(a, x_{0}, x_{1}\right) \stackrel{\text { def }}{=} \Gamma\left(a, x_{0}\right)-\Gamma\left(a, x_{1}\right)$. We also define $\gamma(a, \infty) \stackrel{\text { def }}{=} \lim _{x \rightarrow \infty} \gamma(a, x)=\Gamma(a)$.

Let $Q(a, x) \stackrel{\text { def }}{=} \Gamma(a, x) / \Gamma(a)$. Let $Q^{-1}(a, x)$ be the inverse of $Q(a, x)$ with respect to $x$. Note that $Q(1, x)=\mathrm{e}^{-x}$ and $Q^{-1}(1, x)=\log (1 / x)$.

Let $m$ be the height of a complete binary tree of $n$ nodes, i.e., $m \stackrel{\text { def }}{=}\lfloor\lg n\rfloor$. Let $\ell \stackrel{\text { def }}{=}\lfloor\lg \lg n\rfloor$. Let $L \stackrel{\text { def }}{=}\left\lfloor\left(2-\frac{1}{2 k}\right) \lg \lg n\right\rfloor$.

For node $v \in T_{n}^{\text {bin }}$, let $h(v)$ be the height of $v$, i.e., the distance (number of edges) between $v$ and the root, which we denote by $o$.

Let $\mathcal{X}_{n, y}^{r}$ be $\mathcal{X}_{n}^{r}-1$ conditioned on $T_{k, o}=y$, i.e., the number of $r$-record, excluding the root, conditioned on that the root is removed (cut the $k$-th time) at time $y$.

For functions $f: A \rightarrow \mathbb{R}$ and $g: A \rightarrow \mathbb{R}$, we write $f=O(g)$ uniformly on $B \subseteq A$ to indicate that there exists a constant $C_{0}$ such that $|f(a)| \leqslant C_{0}|g(a)|$ for all $a \in B$. The word uniformly stresses that $C_{0}$ does not depend on $a$.

We use the notation $O_{p}(\cdot)$ and $o_{p}(\cdot)$ in the usual sense, see [14].

The notations $C_{1}(\cdots), C_{2}(\cdots), \ldots$ denote constants that depend on $k$ and other parameters but do not depend on $n$.

\section{The expectation and the variance}

Lemma 3.1. There exist constants $\left(C_{5}(j, b)\right)_{j \geqslant 1, b \geqslant k+1}$ such that

$$
\exp \left(\frac{m x^{k}}{k !}\right) Q(k, x)^{m}=1+\sum_{j=1}^{k} \sum_{b=j k+j}^{j k+k} C_{5}(j, b) m^{j} x^{b}+O\left(m^{k+1} x^{(k+1)^{2}}+m x^{2 k+1}\right)
$$

uniformly for all $x \in\left(0, m^{-k_{0}}\right)$, where $k_{0} \stackrel{\text { def }}{=} \frac{1}{2}\left(\frac{1}{k}+\frac{1}{k+1}\right)$.

Remark 3.1. We do not have a closed form for the constants $C_{5}(j, b)$, but they are the coefficients of $m^{j} x^{b}$ in (3.1). For fixed $k$, they are easy to find with computer algebra systems. For example, when $k=1,(3.1)$ reduces to

$$
\mathrm{e}^{m x} Q(1, x)^{m}=1+O\left(m^{2} x^{4}+m x^{3}\right),
$$


which is trivially true since $Q(1, x)=\mathrm{e}^{-x}$. When $k=2$, (3.1) reduces to

$$
\exp \left(\frac{m x^{2}}{2}\right) Q(2, x)^{m}=1+\frac{1}{3} m x^{3}-\frac{1}{4} m x^{4}+\frac{1}{18} m^{2} x^{6}+O\left(m^{3} x^{9}+m x^{5}\right) .
$$

Proof. Using the series expansion of $Q(k, x)$ given by [6, 8.7.3], it is easy to verify that

$$
\left(\exp \left(\frac{x^{k}}{k !}\right) Q(k, x)\right)^{m}=\left(1-\sum_{j=1}^{k} \frac{x^{k}(-x)^{j}}{(k-1) ! j !(k+j)}-\frac{x^{2 k}}{2(k !)^{2}}+O\left(x^{2 k+1}\right)\right)^{m}
$$

uniformly for $x \in\left(0, m^{-k_{0}}\right)$. Taking the binomial expansion of the right-hand-side and ignoring small order terms gives (3.3).

Lemma 3.2. In the case that the tree is full, i.e., $n=2^{m+1}-1$, then

$$
\mathbb{E} \mathcal{X}_{n, y}^{r}=2^{m+1}\left(\psi_{r}(m, y, 2)+O\left(m^{-\frac{1+r}{k}-1}\right)\right),
$$

where

$$
\begin{aligned}
\psi_{r}(m, z, c) \stackrel{\text { def }}{=} & \frac{m^{-\frac{r}{k}}}{r !}\left(\frac{(k !)^{\frac{r}{k}}}{k} \gamma\left(\frac{r}{k}, \frac{m z^{k}}{k !}\right)+c \frac{(k !)^{\frac{r}{k}}}{k} m^{-1} \gamma\left(\frac{r+k}{k}, \frac{m z^{k}}{k !}\right)\right. \\
& +\sum_{j=1}^{k}\left(\sum_{b=j k+j}^{j k+k} \frac{(k !)^{\frac{b+r}{k}}}{k} C_{6}(j, b) m^{j-\frac{b}{k}} \gamma\left(\frac{b+r}{k}, \frac{m z^{k}}{k !}\right)\right) \\
& \left.+\sum_{i=1}^{k} \frac{(-1)^{i}(k !)^{\frac{i+r}{k}}}{k i !} m^{-\frac{i}{k}} \gamma\left(\frac{i+r}{k}, \frac{m z^{k}}{k !}\right)\right),
\end{aligned}
$$

where the implicit constants $C_{6}(j, b)$ are defined in (3.11).

Proof. Let $v$ be a node of height $i$. For $v$ to be an $r$-record, conditioning on $T_{k, o}=y$, we need $T_{r, v}<y$ and $T_{k, u}>T_{r, v}$ for every $u$ that is an ancestor of $v$. Recall that $T_{r, v} \stackrel{\text { def }}{=} \sum_{j=1}^{r} E_{j, v}$, where $E_{j, v}$ are i.i.d. exponential 1 random variables. Thus $T_{k, u}$ are i.i.d. $\operatorname{Gamma}(k, 1)$ random variables and $T_{r, v}$ is a $\operatorname{Gamma}(r, 1)$ random variable, which are independent from everything else. (See Theorem 2.1.12 of [8] for the relation between exponential distributions and Gamma distributions.)

The Gamma distribution Gamma $(r, 1)$ has the density function

$$
g_{r}(x)= \begin{cases}\frac{x^{r-1} \mathrm{e}^{-x}}{\Gamma(r)} & \text { if } x \geqslant 0 \\ 0 & \text { if } x<0\end{cases}
$$

which implies $\mathbb{P}\{\operatorname{Gamma}(r, 1)>x\}=Q(r, x)$. Thus,

$$
\begin{aligned}
\mathbb{E}\left[I_{r, v} \mid T_{k, o}=y\right] & =\int_{0}^{y} g_{r}(x) \mathbb{P}\{\operatorname{Gamma}(k, 1)>x\}^{i-1} \mathrm{~d} x \\
& =\int_{0}^{y} \frac{x^{r-1} \mathrm{e}^{-x}}{\Gamma(r)} Q(k, x)^{i-1} \mathrm{~d} x .
\end{aligned}
$$


When the tree is full, each level $i$ has $2^{i}$ nodes. Thus in this case

$$
\begin{aligned}
\mathbb{E} \mathcal{X}_{n, y}^{r} & =\sum_{i=1}^{m} 2^{i} \int_{0}^{y} \frac{x^{r-1} \mathrm{e}^{-x}}{\Gamma(r)} Q(k, x)^{i-1} \mathrm{~d} x \\
& =\int_{0}^{y} 2 \frac{x^{r-1} \mathrm{e}^{-x}}{\Gamma(r)}\left(\sum_{i=1}^{m}(2 Q(k, x))^{i-1}\right) \mathrm{d} x \\
& =\frac{2^{m+1}}{r !} \int_{0}^{y} x^{r-1} \mathrm{e}^{-\frac{m x^{k}}{k !}} h_{0}(x)\left(\mathrm{e}^{\frac{x^{k}}{k !}} Q(k, x)\right)^{m} \mathrm{~d} x+O(1),
\end{aligned}
$$

where

$$
h_{0}(x) \stackrel{\text { def }}{=} \frac{\mathrm{e}^{-x}}{(2 Q(k, x)-1)}=1+\frac{2 x^{k}}{k !}+\sum_{i=1}^{k} \frac{(-1)^{i} x^{i}}{i !}+O\left(x^{k+1}\right),
$$

as $x \rightarrow 0$ by [6, 8.7.3]. Thus uniformly for $0<x \leqslant m^{-k_{0}}$ with $k_{0} \stackrel{\text { def }}{=} \frac{1}{2}\left(\frac{1}{k}+\frac{1}{k+1}\right)$,

$$
\begin{aligned}
h_{0}(x)\left(\mathrm{e}^{\frac{x^{k}}{k !}} Q(k, x)\right)^{m}= & +\frac{2 x^{k}}{k !}+\sum_{i=1}^{k} \frac{(-1)^{i} x^{i}}{i !}+\sum_{j=1}^{k}\left(\sum_{b=j k+j}^{j k+k} x^{b} m^{j} C_{6}(j, b)\right) \\
& +O\left(x^{k+1}+m^{k+1} x^{(k+1)^{2}}+m x^{2 k+1}\right)
\end{aligned}
$$

for some constants $C_{6}(j, b)$, where we expand the left-hand-side using (3.10) and Lemma 3.1, and then omit small order terms.

Note that for $b \geqslant 0$ and $j \geqslant 0$,

$$
\int_{0}^{y} \exp \left(-\frac{m x^{k}}{k !}\right) x^{r-1} x^{b} m^{j} \mathrm{~d} x=\frac{(k !)^{\frac{b+r}{k}}}{k} m^{j-\frac{b+r}{k}} \gamma\left(\frac{b+r}{k}, \frac{m y^{k}}{k !}\right) .
$$

Thus if $y<m^{-k_{0}}$, by putting the expansion (3.11) into (3.9) and integrating term by term, we get (3.5).

For $y>m^{-k_{0}}$, it is not difficult to verify that the part of the integral in (3.9) over $\left[m^{-k_{0}}, y\right]$ and the difference $\psi_{r}(m, y, 2)-\psi_{r}\left(m, m^{-k_{0}}, 2\right)$ are both exponentially small and can be absorbed by the error term.

Lemma 3.3. If $h(v)=m$, then

$$
\mathbb{E}\left[I_{r, v} \mid T_{k, o}=y\right]=\psi_{r}(m, y, 1)+O\left(m^{-\frac{1+r}{k}-1}\right)=\psi_{r}^{*}(m, y)+O\left(m^{-\frac{1+r}{k}}\right),
$$

where

$$
\psi_{r}^{*}(m, y) \stackrel{\text { def }}{=} \frac{m^{-\frac{r}{k}}}{r !} \frac{(k !)^{\frac{r}{k}}}{k} \gamma\left(\frac{r}{k}, \frac{m y^{k}}{k !}\right) .
$$

Proof. When $v$ is a node of height $m$, by (3.8),

$$
\begin{aligned}
\mathbb{E}\left[I_{r, v} \mid T_{k, o}=y\right] & =\int_{0}^{y} \frac{x^{r-1} \mathrm{e}^{-x}}{\Gamma(r)} Q(k, x)^{m-1} \mathrm{~d} x \\
& =\frac{1}{\Gamma(r)} \int_{0}^{y} x^{r-1} \frac{x^{r-1} \mathrm{e}^{-x}}{\Gamma(r)} h_{2}(x)\left(\mathrm{e}^{\frac{x^{k}}{k !}} Q(k, x)\right)^{m} \mathrm{~d} x,
\end{aligned}
$$


where $h_{2}(x) \stackrel{\text { def }}{=} \frac{\mathrm{e}^{-x}}{Q(k, x)}$. Expanding $h_{2}(x)$ by $[6,8.7 .3]$ and using Lemma 3.1, we have, uniformly for $x \in\left(0, m^{-k_{0}}\right)$ with $k_{0} \stackrel{\text { def }}{=} \frac{1}{2}\left(\frac{1}{k}+\frac{1}{k+1}\right)$

$$
\begin{aligned}
h_{2}(x)\left(\mathrm{e}^{\frac{x^{k}}{k !}} Q(k, x)\right)^{m}= & +\frac{x^{k}}{k !}+\sum_{i=1}^{k} \frac{(-1)^{i} x^{i}}{i !}+\sum_{j=1}^{k}\left(\sum_{b=j k+j}^{j k+k} x^{b} m^{j} C_{6}(j, b)\right) \\
& +O\left(x^{k+1}+m^{k+1} x^{(k+1)^{2}}+m x^{2 k+1}\right) .
\end{aligned}
$$

Note that this differs from (3.11) only by the constant in the term $x^{k} / k$ !. Thus the first equality in (3.13) follows as in Lemma 3.2. The second equality follows by keeping only the main term of $\psi_{r}(m, y, 1)$.

The next lemma computes $\mathbb{E} \mathcal{X}_{n, y}^{r}$ when the tree is not full. The reason why it is formulated in terms of $\bar{m}$ will be clear in the proof of Lemma 4.2.

Lemma 3.4. Let $\varphi_{r}(n, y) \stackrel{\text { def }}{=} \mathbb{E} \mathcal{X}_{n, y}^{r}$. Let

$$
\begin{aligned}
\bar{\psi}_{r}(n, m, z) & \stackrel{\text { def }}{=} 2^{m+1} \psi_{r}(m, z, 2)-\left(2^{m+1}-n\right) \psi_{r}(m, z, 1) \\
& =n \psi_{r}(m, z, 1)+\frac{(k !)^{\frac{r}{k}}}{k r !} \frac{2^{m+1}}{m^{1+\frac{r}{k}}} \gamma\left(1+\frac{r}{k}, \frac{m z^{k}}{k !}\right)
\end{aligned}
$$

If $2^{\bar{m}}-1 \leqslant n \leqslant 2^{\bar{m}+1}-1$, then

$$
\varphi_{r}(n, y)=\bar{\psi}_{r}(n, \bar{m}, y)+O\left(n \bar{m}^{-\frac{1+r}{k}-1}\right) .
$$

Proof. Assume first that $\bar{m}=m$. When the tree is not necessarily full, the estimate of $\varphi_{r}(n, y)$ in (3.5) over counts the number of nodes at height $m$ by $2^{m+1}-n$. The contribution of the over counted nodes in (3.5) can be estimated using (3.13). Subtracting this part from (3.5) gives (3.18).

The only other possible case is that $\bar{m}=m+1$ and the tree is full. The result follows easily by adding an extra node $v$ at height $\bar{m}$, computing the total expectation of $r$-records for this tree by the case already studied, and subtracting $\mathbb{E}\left[I_{r, v} \mid T_{k, o}=y\right] \sim \psi_{r}(m, y, 1)$ from (3.13).

Corollary 3.1. We have

$$
\mathbb{E} \mathcal{X}_{n}^{r}=\frac{C_{2}(r) n}{\lg (n)^{\frac{r}{k}+1}}\left(\mu_{r, n}-\lg \lg n\right)+\frac{C_{2}(r) 2^{m+1}}{\lg (n)^{\frac{r}{k}+1}}+O\left(n \lg (n)^{-\frac{r+1}{k}-1}\right),
$$

where $\mu_{r, n}$ is defined in (1.3).

Proof. Lemma 3.4 gives an asymptotic expansion of $\varphi_{r}(n, y) \stackrel{\text { def }}{=} \mathbb{E}\left[\mathcal{X}_{n}^{r} \mid T_{k, o}=y\right]$. To get rid of this conditioning, first consider a full binary tree of height $m^{\prime}=m+1$, i.e., a tree of size $n^{\prime}=2^{m+2}-1$. It is easy to see that $\varphi_{r}\left(n^{\prime}, \infty\right)$ is exactly twice of $\mathbb{E} \mathcal{X}_{n}^{r}$ for $n=2^{m+1}-1$. This solves the case when the tree is full. 
The general case can be solved similarly. Consider a binary tree, with the right subtree of the root being $T_{n}^{\text {bin }}$ (possibly not full), and the left subtree of the root being $T_{2^{m+1}-1}^{\text {bin }}$, i.e., a full binary tree of height $m$. This tree has size $n^{\prime \prime}=n+2^{m+1}$. Thus $\varphi_{r}\left(n^{\prime \prime}, \infty\right)$ is the expected number of $r$-records in $T_{n}^{\text {bin }}$, plus the expected number of $r$-record in $T_{2^{m+1}-1}^{\text {bin }}$, which is $\varphi\left(n^{\prime}, \infty\right) / 2$ by the previous paragraph. Thus

$$
\mathbb{E} \mathcal{X}_{n}^{r}=\varphi_{r}\left(n^{\prime \prime}, \infty\right)-\frac{1}{2} \varphi_{r}\left(n^{\prime}, \infty\right)
$$

which implies (3.19) by Lemma 3.4.

Remark 3.2. Comparing (3.19) and (1.2) in Theorem 1.1, we see that $\mathcal{X}_{n}^{r}$ is concentrated well above their means (at a distance of about $\left.n \lg (\lg (n)) / \lg (n)^{1+r / k}\right)$. Thus $\mathbb{P}\left\{\mathcal{X}_{n}^{r}<\mathbb{E} \mathcal{X}_{n}^{r}\right\} \rightarrow 0$. See also Remark 1.4 of [12].

Remark 3.3. The simplest case that $r=k$ and the tree is full can also be computed directly by noticing that

$$
\begin{array}{rlrl}
\mathbb{E} \mathcal{X}_{n}^{k} & =\sum_{v} \frac{1}{h(v)+1}=\sum_{i=0}^{m} \frac{2^{i}}{i+1}=-2^{m+1} \Phi(2,1, m+2)-\frac{1}{2}(\mathrm{i} \pi) & \\
& =\frac{2^{m+1}}{m+2}\left(1+\sum_{n=1}^{N-1}(-1)^{n-1}(m+2)^{-n} \operatorname{Li}_{-n}(2)+O\left(m^{-N}\right)\right) \quad(N \in \mathbb{N}) \\
& =2^{m+1}\left(m+2 m^{-3}+6 m^{-4}+38 m^{-5}+O\left(m^{-6}\right)\right) & (N=5),
\end{array}
$$

where $\Phi(z, s, a)$ denotes Hurwitz-Lerch zeta function [6, 25.14], $\operatorname{Li}_{s}(z)$ denotes the polylogarithm function [6,25.12], and the last step uses an asymptotic expansion of $\Phi(z, s, a)$ given in [4].

Lemma 3.5. We have

$$
\operatorname{Var}\left(\mathcal{X}_{n, y}^{r}\right)=O\left(n^{2} m^{-\frac{2 r+1}{k}}\right)
$$

Proof. Consider two nodes, $v$ and $w$ of heights $s$ and $t$ respectively. Let $u$ be the node that is furthest away from the root among the common ancestor of $v$ and $w$. Let $i=h(u)$.

We call the pair $(v, w)$ good if $i \leqslant \frac{m}{3}$ and $s, t \geqslant \frac{2 m}{3}$. Otherwise we call it bad. Assume for now that $(v, w)$ is good.

Let $o=u_{0}, \ldots, u_{i}=u$ be the path from the root to $u$. Let $Z=\min _{1 \leqslant j \leqslant i} T_{k, u_{i}}$.

Note that conditioning on $T_{k, o}=y$ and $Z=z$, the events that $v$ is an $r$-record and that $w$ is an $r$-record are independent. Thus by Lemma 3.3 and the assumption that $(v, w)$ is good,

$$
\mathbb{E}\left[I_{r, v} I_{r, w} \mid T_{k, o}=y, Z=z\right]=\psi_{r}^{*}(s-i, z \wedge y) \psi_{r}^{*}(t-i, z \wedge y)+O\left(m^{-\frac{2 r+1}{k}}\right),
$$

where $a \wedge b \stackrel{\text { def }}{=} \min \{a, b\}$.

Since $\psi_{r}^{*}(a, w)$ is increasing in $w,(3.23)$ implies that, after averaging over $z$,

$$
\mathbb{E}\left[I_{r, v} I_{r, w} \mid T_{k, o}=y\right] \leqslant \psi_{r}^{*}(s-i, y) \psi_{r}^{*}(t-i, y)+O\left(m^{-\frac{2 r+1}{k}}\right) .
$$


On the other hand, again by Lemma 3.3 and the assumption that $(v, w)$ is good,

$$
\mathbb{E}\left[I_{r, v} \mid T_{k, o}=y\right] \mathbb{E}\left[I_{r, w} \mid T_{k, o}=y\right]=\psi_{r}^{*}(s, y) \psi_{r}^{*}(t, y)+O\left(m^{-\frac{2 r+1}{k}}\right) .
$$

Therefore, by the definition of $\psi_{r}^{*}(a, w)$ in (3.14), the first order term of the above is

$$
\begin{aligned}
\operatorname{Cov}\left(I_{r, v}, I_{r, w} \mid T_{k, o}=y\right) \leqslant & \psi_{r}^{*}(s-i, y) \psi_{r}^{*}(t-i, y)-\psi_{r}^{*}(s, y) \psi_{r}^{*}(s, y)+O\left(m^{-\frac{2 r+1}{k}}\right) \\
= & O\left(m^{-\frac{2 r}{k}}\right)\left(i m^{-1}+\Gamma\left(\frac{r}{k}, \frac{s y^{k}}{\Gamma(k+1)}\right)-\Gamma\left(\frac{r}{k}, \frac{(s-i) y^{k}}{\Gamma(k+1)}\right)\right. \\
& +\Gamma\left(\frac{r}{k}, \frac{t y^{k}}{\Gamma(k+1)}\right)-\Gamma\left(\frac{r}{k}, \frac{(t-i) y^{k}}{\Gamma(k+1)}\right) \\
& +\Gamma\left(\frac{r}{k}, \frac{(s-i) y^{k}}{\Gamma(k+1)}\right) \Gamma\left(\frac{r}{k}, \frac{(t-i) y^{k}}{\Gamma(k+1)}\right) \\
& \left.-\Gamma\left(\frac{r}{k}, \frac{s y^{k}}{\Gamma(k+1)}\right) \Gamma\left(\frac{r}{k}, \frac{t y^{k}}{\Gamma(k+1)}\right)\right) .
\end{aligned}
$$

For $x_{1} \leqslant x_{2}$ and $0 \leqslant a \leqslant 1$,

$$
\Gamma\left(a, x_{1}\right)-\Gamma\left(a, x_{2}\right)=\int_{x_{1}}^{x_{2}} \mathrm{e}^{-x} x^{a-1} \mathrm{~d} x \leqslant \mathrm{e}^{-x_{1}} x_{1}^{a-1}\left(x_{2}-x_{1}\right) \leqslant\left(\frac{a}{\mathrm{e}}\right)^{a} \frac{x_{2}-x_{1}}{x_{1}},
$$

since $\mathrm{e}^{-x} x^{a-1}$ is decreasing and $\mathrm{e}^{-x} x^{a} \leqslant\left(\frac{a}{\mathrm{e}}\right)^{a}$. Thus when $(v, w)$ is good,

$$
\left|\Gamma\left(\frac{r}{k}, \frac{s y^{k}}{\Gamma(k+1)}\right)-\Gamma\left(\frac{r}{k}, \frac{(s-i) y^{k}}{\Gamma(k+1)}\right)\right|=O\left(\frac{i}{m}\right) .
$$

Cancelling other terms in (3.26) in a similar way shows that

$$
\operatorname{Cov}\left(I_{r, v}, I_{r, w} \mid T_{k, o}=y\right)=O\left(m^{-\frac{2 r+1}{k}}+i m^{-1-\frac{2 r}{k}}\right) .
$$

Given $i, s, t$, there are at most $2^{s+t-i}$ choices of $u, v, w$. Thus

$$
\begin{aligned}
& \sum_{\operatorname{good}(v, w)} \operatorname{Cov}\left(I_{r, v}, I_{r, w} \mid T_{k, o}=y\right) \\
& \leqslant \sum_{i=1}^{m} \sum_{s=1}^{m} \sum_{t=1}^{m} 2^{s+t-i} O\left(i m^{-1-\frac{2 r}{k}}+m^{-\frac{2 r+1}{k}}\right)=O\left(n^{2} m^{-\frac{2 r+1}{k}}\right) .
\end{aligned}
$$

The number of bad pairs is at most

$$
\sum_{i>\frac{m}{3}, s, t \leqslant m} 2^{s+t-i}+2 \sum_{i>0, t<\frac{2 m}{3}, s \leqslant m} 2^{s+t-i}=O\left(2^{2 m-\frac{m}{3}}\right)=O\left(n^{\frac{5}{3}}\right) .
$$

Using the fact that $\operatorname{Cov}\left(I_{r, v}, I_{r, w} \mid T_{k, o}=y\right) \leqslant 1$, it follows from (3.30) and (3.31) that

$$
\operatorname{Var}\left(\mathcal{X}_{n, y}^{r}\right)=\sum_{v, w} \operatorname{Cov}\left(I_{r, v}, I_{r, w} \mid T_{k, o}=y\right)=O\left(n^{2} m^{-\frac{2 r+1}{k}}\right)
$$

as the lemma claims. 
Recall that $L \stackrel{\text { def }}{=}\left\lfloor\left(2-\frac{1}{2 k}\right) \lg \lg n\right\rfloor$. Let $\left(v_{i}, 1 \leqslant i \leqslant 2^{L}\right)$ be the $2^{L}$ nodes of height $L$. Let $Y_{i}$ be the minimum of the $T_{k, v}$ for all nodes $v$ on the path between the root and $v_{i}$.

Lemma 3.6. We have

$$
\mathcal{X}_{n}^{r}=\sum_{i=1}^{2^{L}} \varphi_{r}\left(n_{i}, Y_{i}\right)+O_{p}\left(n m^{-1-\frac{1}{4 k}-\frac{r}{k}}\right) .
$$

Proof. The proof uses the estimate of the variance in Lemma 3.5 and exactly the same argument of Lemma 2.3 in [12]. We omit the details.

\section{Transformation into a triangular array}

In this section, we prove Proposition 4.1, which shows that $\mathcal{X}_{n}^{r}$, properly rescaled and shifted, can be written as a sum of independent random variables. Three technical lemmas Lemma 4.1, Lemma 4.2, Lemma 4.3 are needed.

Proposition 4.1. Let $\alpha_{n} \stackrel{\text { def }}{=}\{\lg n\}$ and $\beta_{n} \stackrel{\text { def }}{=}\{\lg \lg n\}$. Then

$$
\begin{aligned}
\frac{m^{\frac{r}{k}+1}}{n C_{2}(r)} \mathcal{X}_{n}^{r} & -\frac{k}{r} \lg (n)-\sum_{i=1}^{k} C_{1}(r, i) \lg (n)^{1-\frac{i}{k}}-\lg (\lg (n)) \\
= & 2^{1-\alpha_{n}}+\alpha_{n}-\beta_{n}-\ell+L+1-C_{3}(r) \sum_{v: h(v) \leqslant L} \xi_{r, v}+o_{p}(1),
\end{aligned}
$$

where

$$
\xi_{r, v} \stackrel{\text { def }}{=} \frac{m n_{v}}{n} \Gamma\left(\frac{r}{k}, \frac{m T_{k, v}^{k}}{k !}\right)
$$

and

$$
\begin{aligned}
& C_{1}(r, i) \stackrel{\text { def }}{=} C_{7}(r, i)+\sum_{j=1}^{i} C_{8}(r, j, j k+i), \\
& C_{2}(r) \stackrel{\text { def }}{=} \frac{(k !)^{r / k} \Gamma\left(\frac{r}{k}\right)}{k^{2} \Gamma(r)}, \quad C_{3}(r) \stackrel{\text { def }}{=} \frac{1}{\Gamma\left(1+\frac{r}{k}\right)}, \\
& C_{7}(r, i) \stackrel{\text { def }}{=} \frac{(-1)^{i} k(k !)^{i / k} \Gamma\left(\frac{i+r}{k}\right)}{r i ! \Gamma\left(\frac{r}{k}\right)}, \quad C_{8}(r, j, b) \stackrel{\text { def }}{=} \frac{k(k !)^{b / k} C_{6}(j, b) \Gamma\left(\frac{b+r}{k}\right)}{r \Gamma\left(\frac{r}{k}\right)} .
\end{aligned}
$$

Proof of Proposition 4.1. Expanding (4.29) in Lemma 4.3 bellow and dividing both sides by $n m^{-\frac{r}{k}-1} C_{2}(r)$ shows that

$$
\begin{aligned}
\frac{m^{\frac{r}{k}+1}}{n C_{2}(r)} \mathcal{X}_{n}^{r}= & \frac{k m}{r}+L+\frac{2^{m+1}}{n}+1+\sum_{i=1}^{k} C_{7}(r, i) m^{1-\frac{i}{k}} \\
& +\sum_{j=1}^{k} \sum_{b=j(k+1)}^{(j+1) k} C_{8}(r, j, b) m^{-\frac{b}{k}+j+1}-C_{3}(r) \sum_{v} \xi_{r, v}+O\left(m^{-\frac{1}{4 k}}\right) .
\end{aligned}
$$


Subtracting

$$
m^{\frac{r}{k}+1} \lg (n)^{-\frac{r}{k}-1}\left(\frac{k}{r} \lg (n)+\sum_{i=1}^{k} C_{1}(r, i) \lg (n)^{1-\frac{i}{k}}+\lg (\lg (n))\right),
$$

from both sides of (4.4) gives (4.1).

Lemma 4.1. Recall that $Y_{1}$ has the distribution of the minimum of $L+1$ independent $\operatorname{Gamma}(k, 1)$ random variables. Let $\hat{m} \stackrel{\text { def }}{=} m-L$. Let $a>0$ be a constant. Then

$$
\begin{array}{lr}
\mathbb{E}\left[\Gamma\left(a, \frac{\hat{m} Y_{1}^{k}}{k !}\right)\right]=O\left(\frac{L}{m}\right) & \text { if } a>0, \\
\mathbb{E}\left[\Gamma\left(a, \frac{\hat{m} Y_{1}^{k}}{k !}, \frac{m Y_{1}^{k}}{k !}\right)\right]=O\left(\frac{L^{2}}{m^{2}}\right) & \text { if } 1>a>0, \\
\mathbb{E}\left[\hat{m}^{-a} \Gamma\left(a, \frac{\hat{m} Y_{1}^{k}}{k !}\right)-m^{-a} \Gamma\left(a, \frac{m Y_{1}^{k}}{k !}\right)\right]=O\left(\frac{L^{2}}{m^{a+2}}\right) & \text { if } 1>a>0 .
\end{array}
$$

Proof. Since

$$
\mathbb{P}\left\{Y_{1}>x\right\}=\mathbb{P}\{\operatorname{Gamma}(k, 1)>x\}^{L+1}=Q(k, x)^{L+1},
$$

the density of $Y_{1}$ is

$$
g_{Y_{1}}(x)= \begin{cases}\frac{(1+L)}{\Gamma(k)} \mathrm{e}^{-x} x^{k-1} Q(k, x)^{L} & \text { if } x \geqslant 0 \\ 0 & \text { if } x<0\end{cases}
$$

by the derivative formula

$$
\frac{\mathrm{d}}{\mathrm{d} z} Q(a, z)=-\frac{z^{a-1} \mathrm{e}^{-z}}{\Gamma(a)}, \quad \frac{\mathrm{d}}{\mathrm{d} x} Q^{-1}(a, x)=-\Gamma(a) \exp \left(Q^{-1}(a, x)\right) Q^{-1}(a, x)^{1-a},
$$

see $[6,8.8 .13]$. For $0<a \leqslant 1$ and $z \geqslant 0$, by the inequality [6, 8.10.11],

$$
\Gamma(a, z) \leqslant \Gamma(a)\left(1-\left(1-\mathrm{e}^{-z}\right)^{a}\right) \leqslant \Gamma(a) \mathrm{e}^{-z} .
$$

Therefore,

$$
\begin{aligned}
\mathbb{E}\left[\Gamma\left(a, \frac{\hat{m} Y_{1}^{k}}{k !}\right)\right] & =\int_{0}^{\infty} g_{Y_{1}}(x) \Gamma\left(a, \frac{\hat{m} x^{k}}{k !}\right) \mathrm{d} x \\
& \leqslant O(L) \int_{0}^{\infty} x^{k-1} \exp \left(-\frac{\hat{m} x^{k}}{k !}\right) \mathrm{d} x=O\left(\frac{L}{m}\right) .
\end{aligned}
$$

For $a>1$ and $z \geqslant 0$, also by [6, 8.10.11],

$$
\begin{aligned}
\Gamma(a, z) & \leqslant \Gamma(a)\left(1-\left(1-\exp \left(-\frac{m \Gamma(a+1)^{-1 / a} x^{k}}{k !}\right)\right)^{a}\right) \\
& \leqslant a \Gamma(a) \exp \left(-\frac{m \Gamma(a+1)^{-1 / a} x^{k}}{k !}\right),
\end{aligned}
$$


where the last inequality follows from that $(1-b)^{a} \geqslant 1-a b$ for $b \in(0,1)$ and $a>1$. Therefore, similar to (4.13),

$$
\mathbb{E}\left[\Gamma\left(a, \frac{\hat{m} Y_{1}^{k}}{k !}\right)\right] \leqslant O(L) \int_{0}^{\infty} x^{k-1} \exp \left(-\frac{m x^{k}}{k !} \Gamma(a+1)^{-\frac{1}{a}}\right) \mathrm{d} x=O\left(\frac{L}{m}\right) .
$$

Thus we have (4.6).

For (4.7), first by (4.10),

$$
\mathbb{E}\left[\Gamma\left(a, \frac{\hat{m} Y_{1}^{k}}{k !}, \frac{m Y_{1}^{k}}{k !}\right)\right]=\int_{0}^{\infty} g_{Y_{1}}(x) \Gamma\left(a, \frac{\hat{m} x^{k}}{k !}, \frac{m x^{k}}{k !}\right) \mathrm{d} x .
$$

Since $\mathrm{e}^{-x} x^{a-1}$ is decreasing when $0<a \leqslant 1$, for $0<x_{1}<x_{2}$

$$
\Gamma\left(a, x_{1}, x_{2}\right)=\int_{x_{1}}^{x_{2}} \mathrm{e}^{-x} x^{a-1} \mathrm{~d} x \leqslant\left(x_{2}-x_{1}\right) \mathrm{e}^{-x_{1}} x_{1}^{a-1}
$$

Therefore,

$$
\Gamma\left(a, \frac{\hat{m} x^{k}}{k !}, \frac{m x^{k}}{k !}\right) \leqslant L \hat{m}^{a-1}(k !)^{-a} x^{a k} \exp \left(-\frac{\hat{m} x^{k}}{k !}\right) .
$$

Substituting the above inequality into (4.16) and integrating gives (4.7).

For (4.8), note that

$$
\begin{aligned}
& \hat{m}^{-a} \Gamma\left(a, \frac{Y_{1}^{k} \hat{m}}{k !}\right)-m^{-a} \Gamma\left(a, \frac{m Y_{1}^{k}}{k !}\right) \\
& =\left(\hat{m}^{-a}-m^{-a}\right) \Gamma\left(a, \frac{\hat{m} Y_{1}^{k}}{k !}\right)+m^{-a} \Gamma\left(a, \frac{Y_{1}^{k} \hat{m}}{k !}, \frac{m Y_{1}^{k}}{k !}\right),
\end{aligned}
$$

where $\Gamma\left(a, x_{0}, x_{1}\right) \stackrel{\text { def }}{=} \Gamma\left(a, x_{0}\right)-\Gamma\left(a, x_{1}\right)$. The result follows easily from (4.6) and (4.7).

The next two lemmas first remove the $\bar{m}$ (see Lemma 3.4) hidden in the representation (3.33) then transform it into a sum of independent random variables.

Lemma 4.2 . Let $n_{i}$ be the size of the subtree rooted at $v_{i}$. Then

$$
\begin{aligned}
\mathcal{X}_{n}^{r}= & \bar{\psi}_{r}(n, m, \infty)+\frac{r(k !)^{r / k} \Gamma\left(\frac{r}{k}\right)}{k^{2} r !} n m^{-\frac{k+r}{k}} L-\sum_{i=1}^{2^{L}} \frac{n_{i}}{k r !}\left(\frac{m}{k !}\right)^{-\frac{r}{k}} \Gamma\left(\frac{r}{k}, \frac{m Y_{i}^{k}}{k !}\right) \\
& +O_{p}\left(n m^{-1-\frac{1}{4 k}-\frac{r}{k}}\right) .
\end{aligned}
$$


Proof. By Lemma 3.4, we have

$$
\begin{aligned}
\varphi_{r}\left(n_{i}, y\right)= & \frac{n_{i}(k !)^{r / k} \hat{m}^{-\frac{r}{k}} \gamma\left(\frac{r}{k}, \frac{\hat{m} Y_{i}^{k}}{k !}\right)}{k r !} \\
& +\frac{n_{i}(k !)^{r / k} \hat{m}^{-\frac{k+r}{k}} \gamma\left(\frac{k+r}{k}, \frac{\hat{m} Y_{i}^{k}}{k !}\right)}{k r !}+\frac{2^{\hat{m}+1}(k !)^{r / k} \hat{m}^{-\frac{k+r}{k}} \gamma\left(\frac{k+r}{k}, \frac{m Y_{i}^{k}}{k !}\right)}{k r !} \\
& +\sum_{j=1}^{k} \sum_{b=j(k+1)}^{(j+1) k} \frac{(k !)^{\frac{b+r}{k}}}{k r !} n_{i} C_{6}(j, b) \hat{m}^{j-\frac{b+r}{k}} \gamma\left(\frac{b+r}{k}, \frac{\hat{m} Y_{i}^{k}}{k !}\right) \\
& +\sum_{i=1}^{k} \frac{(-1)^{i} n_{i}(k !)^{\frac{i+r}{k}} \hat{m}^{-\frac{i+r}{k}} \gamma\left(\frac{i+r}{k}, \frac{\hat{m} Y_{i}^{k}}{k !}\right)}{k i ! r !}+O_{p}\left(n_{i} \hat{m}^{-\frac{k+r+1}{k}}\right),
\end{aligned}
$$

where $\hat{m}=m-L=m-O(\log m)$. (This is why we need to formulate Lemma 3.4 in terms of $\bar{m}$-here $\hat{m}$ is either the height of subtree rooted at $v_{i}$, or it is the height of the subtree plus one and the subtree is full.)

We now convert this into an expression in $m$. Let

$$
\begin{aligned}
x_{i}= & \frac{n_{i}(k !)^{r / k} m^{-\frac{r}{k}} \Gamma\left(\frac{r}{k}\right)}{k r !}-\frac{n_{i}(k !)^{r / k} m^{-\frac{r}{k}} \Gamma\left(\frac{r}{k}, \frac{m Y_{i}^{k}}{k !}\right)}{k r !} \\
& +\frac{n_{i}(k !)^{r / k} m^{-\frac{k+r}{k}} \Gamma\left(\frac{k+r}{k}\right)}{k r !}+\frac{2^{\hat{m}+1}(k !)^{r / k} m^{-\frac{k+r}{k}} \Gamma\left(\frac{k+r}{k}\right)}{k r !} \\
& +\sum_{j=1}^{k} \sum_{b=j(k+1)}^{(j+1) k} \frac{(k !)^{\frac{b+r}{k}}}{k r !} n_{i} C_{6}(j, b) m^{j-\frac{b+r}{k}} \Gamma\left(\frac{b+r}{k}\right) \\
& +\sum_{i=1}^{k} \frac{(-1)^{i} n_{i}(k !)^{\frac{i+r}{k}} m^{-\frac{i+r}{k}} \Gamma\left(\frac{i+r}{k}\right)}{k i ! r !} .
\end{aligned}
$$

Then using the identity $\gamma(a, z)=\Gamma(a)-\Gamma(a, z)$,

$$
\begin{aligned}
\varphi_{i}\left(n_{i}, y\right)-x_{i}= & \frac{n_{i}(k !)^{r / k}\left(\hat{m}^{-\frac{r}{k}}-m^{-\frac{r}{k}}\right) \Gamma\left(\frac{r}{k}\right)}{k r !} \\
& +\frac{n_{i}(k !)^{r / k}\left(m^{-\frac{r}{k}} \Gamma\left(\frac{r}{k}, \frac{m Y_{i}^{k}}{k !}\right)-\hat{m}^{-\frac{r}{k}} \Gamma\left(\frac{r}{k}, \frac{\hat{m} Y_{i}^{k}}{k !}\right)\right)}{k r !} \\
& +\frac{n_{i}(k !)^{r / k}\left(\hat{m}^{-\frac{k+r}{k}}-m^{-\frac{k+r}{k}}\right) \Gamma\left(\frac{k+r}{k}\right)}{k r !} \\
& +\frac{n_{i}(k !)^{r / k} \hat{m}^{-\frac{k+r}{k}} \Gamma\left(\frac{k+r}{k}, \frac{\hat{m} Y_{i}^{k}}{k !}\right)}{k r !} \\
& +\frac{2^{\hat{m}+1}(k !)^{r / k}\left(\hat{m}^{-\frac{k+r}{k}}-m^{-\frac{k+r}{k}}\right) \Gamma\left(\frac{k+r}{k}\right)}{k r !}
\end{aligned}
$$




$$
\begin{aligned}
& +\frac{2^{\hat{m}+1}(k !)^{r / k} \hat{m}^{-\frac{k+r}{k}} \Gamma\left(\frac{k+r}{k}, \frac{\hat{m} Y_{i}^{k}}{k !}\right)}{k r !} \\
& +\sum_{j=1}^{k} \sum_{b=j(k+1)}^{(j+1) k} \frac{(k !)^{\frac{b+r}{k}}}{k r !} n_{i} C_{6}(j, b)\left(\hat{m}^{j-\frac{b+r}{k}}-m^{j-\frac{b+r}{k}}\right) \Gamma\left(\frac{b+r}{k}\right) \\
& +\sum_{j=1}^{k} \sum_{b=j(k+1)}^{(j+1) k} \frac{(k !)^{\frac{b+r}{k}}}{k r !} n_{i} C_{6}(j, b) \hat{m}^{j-\frac{b+r}{k}} \Gamma\left(\frac{b+r}{k}, \frac{\hat{m} Y_{i}^{k}}{k !}\right) \\
& +\sum_{i=1}^{k} \frac{(-1)^{i} n_{i}(k !)^{\frac{i+r}{k}}\left(\hat{m}^{-\frac{i+r}{k}}-m^{-\frac{i+r}{k}}\right) \Gamma\left(\frac{i+r}{k}\right)}{k i ! r !} \\
& +\sum_{i=1}^{k} \frac{(-1)^{i} n_{i}(k !)^{\frac{i+r}{k}} \hat{m}^{-\frac{i+r}{k}} \Gamma\left(\frac{i+r}{k}, \frac{\hat{m} Y_{i}^{k}}{k !}\right)}{k i ! r !}+O_{p}\left(n_{i} m^{-\frac{k+r+1}{k}}\right) .
\end{aligned}
$$

The first term of the above expression is

$$
\frac{n_{i}(k !)^{r / k}\left(\hat{m}^{-\frac{r}{k}}-m^{-\frac{r}{k}}\right) \Gamma\left(\frac{r}{k}\right)}{k r !}=\frac{r(k !)^{r / k} \Gamma\left(\frac{r}{k}\right) n_{i} m^{-\frac{r}{k}-1} L}{k^{2} r !}+O\left(\frac{n_{i} L^{2}}{m^{\frac{r}{k}+2}}\right),
$$

since $\hat{m}^{-a}-m^{a}=a L m^{-a-1}+O\left(L^{2} m^{-a-2}\right)$. The terms which do not contain $Y_{i}$ can be bounded similarly. For terms involving $Y_{i}$, we can use Lemma 4.1. For example, by (4.8), the second term is

$$
\frac{n_{i}(k !)^{r / k}\left(m^{-\frac{r}{k}} \Gamma\left(\frac{r}{k}, \frac{m Y_{i}^{k}}{k !}\right)-\hat{m}^{-\frac{r}{k}} \Gamma\left(\frac{r}{k}, \frac{\hat{m} Y_{i}^{k}}{k !}\right)\right)}{k r !}=O_{p}\left(n_{i} m^{-\frac{r}{k}-2} L^{2}\right) .
$$

In the end, it follows from Lemma 4.1 and simple asymptotic computations that

$$
\phi_{r}\left(n_{i}, y\right)-x_{i}=\frac{r(k !)^{r / k} \Gamma\left(\frac{r}{k}\right) n_{i} m^{-r / k-1} L}{k^{2} r !}+O_{p}\left(L^{2} n_{i} m^{-\frac{r+1}{k}-1}\right) .
$$

Since $\sum_{i=1}^{2^{L}} n_{i}=n-\left(2^{L}-1\right)=n-O\left(m^{2-\frac{1}{2 k}}\right)$,

$$
\sum_{i}^{2^{L}}\left(\phi_{r}\left(n_{i}, y\right)-x_{i}\right)=\frac{r(k !)^{r / k} \Gamma\left(\frac{r}{k}\right) n m^{-r / k-1} L}{k^{2} r !}+O_{p}\left(L^{2} n m^{-\frac{r+1}{k}-1}\right) .
$$

Thus by (3.33), we have

$$
\begin{aligned}
\mathcal{X}_{n}^{r} & =\sum_{i=1}^{2^{L}} \varphi_{r}\left(n_{i}, y\right)+O_{p}\left(n m^{-1-\frac{1}{4 k}-\frac{r}{k}}\right) \\
& =\sum_{i}^{2^{L}} x_{i}+\frac{r(k !)^{r / k} \Gamma\left(\frac{r}{k}\right) n m^{-r / k-1} L}{k^{2} r !}+O_{p}\left(n m^{-1-\frac{1}{4 k}-\frac{r}{k}}\right),
\end{aligned}
$$

from which (4.20) follows immediately. 
Lemma 4.3. Let $n_{v}$ be the size of the subtree rooted at the node $v$. Then

$$
\begin{aligned}
\mathcal{X}_{n}^{r}= & \bar{\psi}_{r}(n, m, \infty)+\frac{r(k !)^{r / k} \Gamma\left(\frac{r}{k}\right)}{k^{2} r !} n m^{-\frac{k+r}{k}} L \\
& -\sum_{v: h(v) \leqslant L} \frac{n_{v}}{k r !}\left(\frac{m}{k !}\right)^{-\frac{r}{k}} \Gamma\left(\frac{r}{k}, \frac{m T_{k, v}^{k}}{k !}\right)+O_{p}\left(n m^{-1-\frac{1}{4 k}-\frac{r}{k}}\right) .
\end{aligned}
$$

Proof. Recall that $Y_{i}$ is the minimum of $L+1$ independent $\operatorname{Gamma}(k, 1)$ random variables $\left(T_{k, v}, v \in P\left(v_{i}\right)\right)$, where $P\left(v_{i}\right)$ denotes the path from the root $o$ to $v_{i}$. Let $a=$ $(2 k ! \log (m) / m)^{1 / k}$. The probability that at least two $T_{k, v}$ are less than $a$ is

$$
\begin{aligned}
& 1-\mathbb{P}\{\operatorname{Gamma}(k, 1)>a\}^{L+1}-L \mathbb{P}\{\operatorname{Gamma}(k, 1)>a\}^{L} \mathbb{P}\{\operatorname{Gamma}(k, 1) \leqslant a\} \\
& =1-Q(k, x)^{L+1}-L Q(k, x)^{L}(1-Q(k, x)) \\
& =O\left(a^{2 k} L^{2}\right)=O\left(\log (m)^{2} m^{-2} L^{2}\right),
\end{aligned}
$$

where we use the approximation of $Q(k, x)^{L}$ in (3.1) and the series expansion of $Q(k, x)$ in $[6,8.7 .3]$. Thus the probability that this happens for some $i$ is $O\left(2^{L} \log (m)^{2} m^{-2} L^{2}\right)=$ $o(1)$.

With probability goes to 1 , there is at most one $T_{k, v}$ that is less than $a$ on each path $P\left(v_{i}\right)$. When this happens, by the inequality (4.12),

$$
0 \leqslant \sum_{v \in P\left(v_{i}\right)} \Gamma\left(\frac{r}{k}, \frac{m T_{k, v}^{k}}{k !}\right)-\Gamma\left(\frac{r}{k}, \frac{m Y_{i}^{k}}{k !}\right) \leqslant L \Gamma\left(\frac{r}{k}, \frac{m a^{k}}{k !}\right)=O\left(m^{-2} L\right) .
$$

Therefore,

$$
\begin{aligned}
\sum_{i=1}^{2^{L}} n_{i} \Gamma\left(\frac{r}{k}, \frac{m Y_{i}^{k}}{k !}\right) & =\sum_{i=1}^{2^{L}} n_{i} \sum_{v \in P\left(v_{i}\right)} \Gamma\left(\frac{r}{k}, \frac{m T_{k, v}^{k}}{k !}\right)+O\left(n m^{-2} L\right) \\
& =\sum_{h(v) \leqslant L} \Gamma\left(\frac{r}{k}, \frac{m T_{k, v}^{k}}{k !}\right) \sum_{i: v \in P\left(v_{i}\right)} n_{i}+O\left(n m^{-2} L\right) \\
& =\sum_{h(v) \leqslant L} \Gamma\left(\frac{r}{k}, \frac{m T_{k, v}^{k}}{k !}\right) n_{v}+O\left(n m^{-2} L\right),
\end{aligned}
$$

where in the last step we use $n_{v}-2^{L} \leqslant \sum_{i: v \in P\left(v_{i}\right)} n_{i} \leqslant n$. Thus

$$
\sum_{i=1}^{2^{L}} \frac{n_{i}\left(\frac{m}{k !}\right)^{-\frac{r}{k}}}{k r !} \Gamma\left(\frac{r}{k}, \frac{m Y_{i}^{k}}{k !}\right)=\sum_{h(v) \leqslant L} \frac{n_{v}\left(\frac{m}{k !}\right)^{-\frac{r}{k}}}{k r !} \Gamma\left(\frac{r}{k}, \frac{m T_{k, v}^{k}}{k !}\right)+O\left(n m^{-\frac{r}{k}-2} L\right) .
$$

The lemma follows by putting this into (4.20). 


\section{Convergence of the triangular array}

By taking subsequences, we can assume that $\alpha_{n} \stackrel{\operatorname{def}}{=}\{\lg n\} \rightarrow \alpha$ and $\beta_{n} \stackrel{\text { def }}{=}\{\lg \lg n\} \rightarrow \beta$, as $n \rightarrow \infty$ Thus $\lg n=m+\alpha+o(1), \lg m=\lg \lg n+o(1)=l+\beta+o(1)$, where $l \stackrel{\text { def }}{=}\lfloor\lg \lg n\rfloor$. Moreover, $\lg n-\lg \lg n=m-l+\alpha-\beta+o(1)$ and

$$
\{\lg n-\lg \lg n\} \rightarrow \gamma= \begin{cases}\alpha-\beta & \text { if } \alpha>\beta, \\ \alpha-\beta+1 & \text { if } \alpha<\beta, \\ 0 \text { or } 1 & \text { if } \alpha=\beta,\end{cases}
$$

which implies $\gamma \equiv \alpha-\beta(\bmod 1)$.

Lemma 5.1. Let $h \stackrel{\text { def }}{=} 2^{\beta-\alpha} \Gamma\left(\frac{r}{k}\right)$. Assume that $\alpha_{n} \rightarrow \alpha$ and $\beta_{n} \rightarrow \beta$. Then as $n \rightarrow \infty$ :

(i) For all fixed $x>0, \sup _{v} \mathbb{P}\left\{\xi_{r, v}>x\right\} \rightarrow 0$.

(ii) For all fixed $x>0, \sum_{v: h(v) \leqslant L} \mathbb{P}\left\{\xi_{r, v}>x\right\} \rightarrow \nu_{r, k, \gamma}(x, \infty)$, where $\nu_{r, k, \gamma}$ is defined in $(1.5)$

(iii) We have

$$
\begin{aligned}
& \sum_{v: h(v) \leqslant L} \mathbb{E}\left[\xi_{r, v} \mathbb{1}\left[\xi_{r, v} \leqslant h\right]\right]-\Gamma\left(1+\frac{r}{k}\right)\left(2^{1-\alpha}+\alpha-\beta-\ell+L\right) \\
& \rightarrow f_{r, k, \gamma}-\int_{h}^{1} x \mathrm{~d} \nu_{r, k, \gamma}(x),
\end{aligned}
$$

where $f_{r, k, \gamma}$ is a constant defined later in (5.39).

(iv) We have

$$
\sum_{v: h(v) \leqslant L} \operatorname{Var}\left(\xi_{r, v} \mathbb{1}\left[\xi_{r, v} \leqslant h\right]\right) \rightarrow \int_{0}^{h} x^{2} \mathrm{~d} \nu_{r, k, \gamma}(x) .
$$

Before getting into the somewhat complicated proof of Lemma 5.1, we first show why Theorem 1.1 and Theorem 1.2 follow from it.

Let $\xi_{i}^{\prime} \stackrel{\text { def }}{=} \Gamma\left(1+\frac{r}{k}\right)\left(2^{1-\alpha}+\alpha-\beta-\ell+L\right) / n$, which are deterministic. It follows from Lemma 5.1 that we can apply Theorem 15.28 in [15] with $a=0, b=f_{r, k, \gamma}$ to show that the triangular array $\sum_{h(v) \leqslant L} \xi_{r, v}+\sum_{i=1}^{n} \xi_{i}^{\prime}$ converges in distribution to $W_{r, k, \gamma}$ (defined in Theorem 1.1). Thus by Proposition 4.1, Theorem 1.1 follows immediately.

For Theorem 1.2, note that the right-hand-side of (1.6) equals

$$
\begin{aligned}
& \frac{\lg (n)^{\frac{1}{k}+1}}{C_{2}(1) n}\left(\mathcal{X}_{n}-\sum_{r=1}^{k} \frac{C_{2}(r) n}{\lg (n)^{\frac{r}{k}+1}} \mu_{r, n}\right) \\
& =\left(\frac{\lg (n)^{\frac{1}{k}+1}}{C_{2}(1) n} \mathcal{X}_{n}^{1}-\mu_{1, n}\right)+\sum_{r=2}^{k} \frac{C_{2}(r)}{C_{2}(1) \lg (n)^{\frac{r-1}{k}}}\left(\frac{\lg (n)^{\frac{r}{k}+1}}{C_{2}(r) n} \mathcal{X}_{n}^{r}-\mu_{r, n}\right) \\
& =\left(\frac{\lg (n)^{\frac{1}{k}+1}}{C_{2}(1) n} \mathcal{X}_{n}^{1}-\mu_{1, n}\right)+o_{p}(1) \stackrel{d}{\rightarrow} 1-C_{3}(1) W_{1, k, \gamma},
\end{aligned}
$$


where the last two steps follow from Theorem 1.1.

It remains to show that the result for $\mathcal{X}_{n}$ holds for $\mathcal{X}_{n}^{e}$. By identifying cutting an edge with cutting its lower (closer to root) endpoint node, we see that $\mathcal{X}_{n}^{e}$ is distributed as $\mathcal{X}_{n}-k$ conditioned on $T_{k, o}=\infty$. Thus to apply the same argument for $\mathcal{X}_{n}$ to $\mathcal{X}_{n}^{e}$, we only need to make $Y_{i}$ the minimum of $L$, instead of $L+1$, independent $\operatorname{Gamma}(k, 1)$ random variables, and to exclude $\xi_{r, o}$, i.e., to exclude the root, in the sum of the triangular array in (4.1). This minor change certainly does not matter. See also the argument for $k=1$ at the end of [12].

\section{$5.1 \quad$ Proof of Lemma 5.1 (i)}

Recall that in (4.2) we define

$$
\xi_{r, v} \stackrel{\text { def }}{=} \frac{m n_{v}}{n} \Gamma\left(\frac{r}{k}, \frac{m T_{k, v}^{k}}{k !}\right)
$$

where $\left(T_{k, v}, v \in T_{n}^{\text {bin }}\right)$ are i.i.d. $\operatorname{Gamma}(k, 1)$ random variables. Thus $\mathbb{P}\left\{T_{k, v}>x\right\}=$ $Q(k, x)$, where $Q(k, x) \stackrel{\text { def }}{=} \Gamma(k, x) / \Gamma(k)$, see (3.7). Assume for now that $\frac{n x}{\Gamma(1 / k) m n_{v}} \leqslant 1$. Then, for all fixed $x>0$.

$$
\begin{aligned}
\mathbb{P}\left\{\xi_{r, v}>x\right\} & =\mathbb{P}\left\{\Gamma\left(\frac{r}{k}, \frac{m T_{k, v}^{k}}{k !}\right)>\frac{n x}{m n_{v}}\right\} \\
& =\mathbb{P}\left\{T_{k, v} \leqslant\left(\frac{k !}{m} Q^{-1}\left(\frac{r}{k}, \frac{n x}{\Gamma\left(\frac{r}{k}\right) m n_{v}}\right)\right)^{\frac{1}{k}}\right\} \\
& =1-Q\left(k,\left(\frac{k !}{m} Q^{-1}\left(\frac{r}{k}, \frac{n x}{\Gamma\left(\frac{r}{k}\right) m n_{v}}\right)\right)^{1 / k}\right) .
\end{aligned}
$$

The function $Q^{-1}(a, z)$ is only defined for $z \in(0,1]$. However, we can extend its domain to $(0, \infty)$ by letting $Q^{-1}(a, z)=0$ for $z>1$. This extension makes (5.6) also valid for $\frac{n x}{\Gamma(1 / k) m n_{v}}>1$, since in this case every expression in (5.6) equals 0 .

By $[6,8.10 .11]$, for $z \geqslant 0$,

$$
1-\left(1-\exp \left(-\Gamma\left(1+\frac{r}{k}\right)^{-k / r} z\right)\right)^{r / k} \leqslant Q\left(\frac{r}{k}, z\right) \leqslant 1-\left(1-\mathrm{e}^{-z}\right)^{r / k} .
$$

Letting $y=Q\left(\frac{r}{k}, z\right) \in(0,1],(5.7)$ implies that

$$
Q^{-1}\left(\frac{r}{k}, y\right)=z \leqslant \log _{+}\left(\frac{1}{1-(1-y)^{k / r}}\right) \leqslant \log _{+}\left(\frac{1}{y}\right)
$$

where $\log _{+}(z) \stackrel{\text { def }}{=} \max \{\log (z), 0\}$. Similarly, it follows from (5.7) that

$$
Q^{-1}\left(\frac{r}{k}, y\right) \geqslant \Gamma\left(1+\frac{r}{k}\right)^{k / r} \log \left(\frac{r}{k y}\right) \text {. }
$$


Note that (5.8) and (5.9) also hold for $y>1$ by our extension of $Q^{-1}(a, z)$. Thus uniformly for all $v$ with $h(v) \leqslant L$,

$$
\frac{k !}{m} Q^{-1}\left(\frac{r}{k}, \frac{n x}{\Gamma\left(\frac{r}{k}\right) m n_{v}}\right) \leqslant \frac{k !}{m} \log _{+}\left(\frac{\Gamma\left(\frac{r}{k}\right) m n_{v}}{n x}\right)=O\left(\frac{\log _{+} \frac{m}{x}}{m}\right),
$$

where the last step uses that $n_{v} \leqslant n$. Thus we can apply the series expansion of $Q(k, z)$ near $z=0$ in $[6,8.7 .3]$ to $(5.6)$ to get

$$
\begin{aligned}
\mathbb{P}\left\{\xi_{r, v}>x\right\} & =\frac{1}{m} Q^{-1}\left(\frac{r}{k}, \frac{n x}{\Gamma\left(\frac{r}{k}\right) m n_{v}}\right)\left(1+O\left(\frac{1}{m} Q^{-1}\left(\frac{r}{k}, \frac{n x}{\Gamma\left(\frac{r}{k}\right) m n_{v}}\right)\right)^{\frac{1}{k}}\right) \\
& =\frac{1}{m} Q^{-1}\left(\frac{r}{k}, \frac{n x}{\Gamma\left(\frac{r}{k}\right) m n_{v}}\right)\left(1+O\left(\frac{\log _{+} \frac{m}{x}}{m}\right)^{\frac{1}{k}}\right)=O\left(\frac{\log _{+} \frac{m}{x}}{m}\right) .
\end{aligned}
$$

Therefore this probability tends to zero for all fixed $x$.

\subsection{Proof of Lemma 5.1 (ii)}

We reuse the notion of good and bad nodes defined in [12, pp. 250]. A good node $v$ has $n_{v}=2^{m-t}-1$ for some $t$ with $l / 2 \leqslant t \leqslant L$. All other nodes with height at most $L$ are called bad. Janson [12, eq. 20] showed that

$$
\#\left\{v \text { good }: n_{v}=2^{m-t}-1\right\}= \begin{cases}2^{t+\alpha_{n}}+O(1) & \frac{l}{2} \leqslant t<L, \\ \left(2-2^{\alpha_{n}}\right) 2^{L}+O(1) & t=L,\end{cases}
$$

and that the number of bad nodes is $O\left(L+2^{l / 2}\right)=O\left(\mathrm{~m}^{1 / 2}\right)$.

As we have shown in (5.11) that $\mathbb{P}\left\{\xi_{r, v}>x\right\}=O\left(m^{-1} \log _{+} \frac{m}{x}\right)$. By the same argument as in [12, Eq. 21, 22], the bad nodes can be ignored in the proof of (ii), (iii) and (iv) of Lemma 5.1.

Note that for $t \geqslant L, m 2^{m-t} \leqslant 2^{l+1+m-L}<\frac{n x}{\Gamma(r / k)}$ for $n$ large enough, which implies $Q^{-1}\left(\frac{1}{k}, \frac{n x}{\Gamma(r / k) m n_{v}}\right)=0$ by our extension of $Q^{-1}(a, z)$. Thus, it follows from (5.11) and (5.12) that

$$
\begin{aligned}
\sum_{v \text { good }} \mathbb{P}\left\{\xi_{r, v}>x\right\} & \sim \sum_{t \geqslant l / 2}\left(2^{t+\alpha_{n}}+O(1)\right) \frac{1}{m} Q^{-1}\left(\frac{r}{k}, \frac{n x}{\Gamma\left(\frac{r}{k}\right) m n_{v}}\right)+o(1) \\
& =\sum_{t \geqslant l / 2} 2^{t+\alpha_{n}-l-\beta_{n}} Q^{-1}\left(\frac{r}{k}, 2^{t-l+\alpha_{n}-\beta_{n}+o(1)} \frac{x}{\Gamma\left(\frac{r}{k}\right)}\right)+o(1) \\
& =\sum_{i \leqslant l / 2} 2^{-i+\alpha-\beta} Q^{-1}\left(\frac{r}{k}, 2^{-i+\alpha-\beta+o(1)} \frac{x}{\Gamma\left(\frac{r}{k}\right)}\right)+o(1)
\end{aligned}
$$




$$
\rightarrow F_{r}(x) \stackrel{\text { def }}{=} \sum_{-\infty}^{\infty} 2^{-i+\alpha-\beta} Q^{-1}\left(\frac{r}{k}, 2^{-i+\alpha-\beta} \frac{x}{\Gamma\left(\frac{r}{k}\right)}\right) .
$$

(By the inequality $(5.8)$, the function $F_{r}(x)$ is well-defined on $(0, \infty)$.)

Let $j(x) \stackrel{\text { def }}{=}\left\lfloor\lg \left(x / \Gamma\left(\frac{r}{k}\right)\right)+\alpha-\beta\right\rfloor$. Then $2^{j(x)+\beta-\alpha} \leqslant x / \Gamma\left(\frac{r}{k}\right)<2^{j(x)+\beta-\alpha-1}$. In other words $Q^{-1}\left(\frac{r}{k}, 2^{-i+\alpha-\beta} x / \Gamma\left(\frac{r}{k}\right)\right)=0$ for $i \leqslant j(x)$. Thus

$$
\begin{aligned}
F_{r}(x) & =\sum_{i \geqslant j(x)+1} 2^{-i+\alpha-\beta} Q^{-1}\left(\frac{r}{k}, 2^{-i+\alpha-\beta} \frac{x}{\Gamma\left(\frac{r}{k}\right)}\right) \\
& =\sum_{s \geqslant 1} 2^{-s-j(x)+\alpha-\beta} Q^{-1}\left(\frac{r}{k}, 2^{-s-j(x)+\alpha-\beta} \frac{x}{\Gamma\left(\frac{r}{k}\right)}\right) \\
& =\sum_{s \geqslant 1} x^{-1} \Gamma\left(\frac{r}{k}\right) 2^{\left\{\alpha-\beta+\lg \left(x / \Gamma\left(\frac{r}{k}\right)\right)\right\}-s} Q^{-1}\left(\frac{r}{k}, 2^{\left\{\alpha-\beta+\lg \left(x / \Gamma\left(\frac{r}{k}\right)\right)\right\}-s}\right) \\
& =\sum_{s \geqslant 1} x^{-1} \Gamma\left(\frac{r}{k}\right) 2^{\left\{\gamma+\lg \left(x / \Gamma\left(\frac{r}{k}\right)\right)\right\}-s} Q^{-1}\left(\frac{r}{k}, 2^{\left\{\gamma+\lg \left(x / \Gamma\left(\frac{r}{k}\right)\right)\right\}-s}\right),
\end{aligned}
$$

where the last step uses (5.1). Note that $F_{r}(x)$ is continuous and decreasing on $(0, \infty)$, with $F_{r}(x) \rightarrow 0$ as $x \rightarrow \infty$. By the derivative formula (4.11), the derivative of $F_{r}(x)$ is

$$
\frac{\mathrm{d} F_{r}(x)}{\mathrm{d} x}=-\sum_{s \geqslant 1} g_{r}(s, x),
$$

where

$$
\begin{aligned}
g_{r}(s, x) \stackrel{\text { def }}{=} & \frac{\Gamma\left(\frac{r}{k}\right)^{2}}{x^{2}} 4^{\left\{\gamma+\lg \left(x / \Gamma\left(\frac{r}{k}\right)\right)\right\}-s} \exp \left(Q^{-1}\left(\frac{r}{k}, 2^{\left\{\gamma+\lg \left(x / \Gamma\left(\frac{r}{k}\right)\right)\right\}-s}\right)\right) \\
Q^{-1}\left(\frac{r}{k}, 2^{\left\{\gamma+\lg \left(x / \Gamma\left(\frac{r}{k}\right)\right)\right\}-s}\right)^{1-\frac{r}{k}} &
\end{aligned}
$$

Comparing with (1.5), we see that

$$
\frac{\mathrm{d} \nu_{r, k, \gamma}}{\mathrm{d} x}=\sum_{s \geqslant 1} g_{r}(s, x) \leqslant O\left(2^{\left\{\gamma+\lg \left(x / \Gamma\left(\frac{r}{k}\right)\right\}\right.} x^{-2}\right),
$$

and $F_{r}(x)=\nu_{r, k, \gamma}(x, \infty)$, where the inequality follows from (5.8). Thus Lemma 5.1 (ii) is proved.

\subsection{Proof of Lemma 5.1 (iii)}

Assume for now that $h \stackrel{\text { def }}{=} 2^{\beta-\alpha} \Gamma\left(\frac{r}{k}\right)<1$. Let $j_{1}=\left\lfloor\alpha-\beta-\lg \Gamma\left(\frac{r}{k}\right)\right\rfloor$, i.e., $2^{j_{1}} h \leqslant 1<$ $2^{j_{1}+1}$. By the upper bound of $\mathrm{d} \nu_{r, k, \gamma} / \mathrm{d} x=\sum_{s \geqslant 1} g_{r}(s, x)$ in $(5.17), \int_{h}^{1} x \mathrm{~d} \nu_{r, k, \gamma}(x)<\infty$. Thus we are allowed to write this integral as

$$
\int_{h}^{1} x \mathrm{~d} \nu_{r, k, \gamma}(x)=\sum_{i=0}^{j_{1}} \int_{h 2^{i}}^{h 2^{i+1} \wedge 1} x \mathrm{~d} \nu_{r, k, \gamma}(x)=\sum_{s \geqslant 1} \sum_{i=0}^{j_{1}} \int_{h 2^{i}}^{h 2^{i+1} \wedge 1} x g_{r}(s, x) \mathrm{d} x .
$$


For $x \in\left(2^{i} h, 2^{i+1} h\right)$, by the definition of $g_{r}(s, x)$ in $(5.16)$,

$$
\begin{aligned}
& g_{r}(s, x)=\hat{g}_{r}(s, x, i) \stackrel{\text { def }}{=} 4^{-i-s+\alpha-\beta} \\
& \exp \left\{Q^{-1}\left(\frac{r}{k}, 2^{-i-s+\alpha-\beta} \frac{x}{\Gamma\left(\frac{r}{k}\right)}\right)\right\} Q^{-1}\left(\frac{r}{k}, 2^{-i-s+\alpha-\beta} \frac{x}{\Gamma\left(\frac{r}{k}\right)}\right)^{1-\frac{r}{k}} .
\end{aligned}
$$

Using the derivative formula (4.11), one can verify that

$$
\frac{\partial}{\partial x} \hat{G}(s, x, i)=x \hat{g}_{r}(s, x, i)
$$

where

$$
\begin{aligned}
\hat{G}(s, x, i) \stackrel{\text { def }}{=} & \Gamma\left(1+\frac{r}{k}, Q^{-1}\left(\frac{r}{k}, \frac{2^{-i-t+\alpha-\beta} x}{\Gamma\left(\frac{r}{k}\right)}\right)\right) \\
& -x 2^{\alpha-\beta-i-t} Q^{-1}\left(\frac{r}{k}, \frac{2^{-i-t+\alpha-\beta} x}{\Gamma\left(\frac{r}{k}\right)}\right) .
\end{aligned}
$$

Therefore,

$$
\int_{h 2^{i}}^{h 2^{i+1}} x g_{r}(s, x) \mathrm{d} x=\int_{h 2^{i}}^{h 2^{i+1}} x \hat{g}_{r}(s, x, i) \mathrm{d} x=\hat{G}\left(s, h 2^{i+1}, i\right)-\hat{G}\left(s, h 2^{i}, i\right) .
$$

Summing (5.22) over $i$ and $s$ as in (5.18) and simplifying through [6, 8.8.2]

$$
\Gamma(a+1, z)=a \Gamma(a, z)+z^{a} \mathrm{e}^{-z}
$$

we have

$$
\int_{h}^{1} x \mathrm{~d} \nu_{r, k, \gamma}(x)=\Gamma\left(1+\frac{1}{k}\right) j_{1}+\mu\left(\left\{\gamma-\lg \Gamma\left(\frac{r}{k}\right)\right\}\right)-\mu(0)
$$

where

$$
\begin{aligned}
\mu(x) \stackrel{\text { def }}{=} & 2^{x} \Gamma\left(1+\frac{r}{k}\right)+\sum_{s \geqslant 1} \exp \left(-Q^{-1}\left(\frac{r}{k}, 2^{x-s}\right)\right) Q^{-1}\left(\frac{r}{k}, 2^{x-s}\right)^{r / k} \\
& -\sum_{s \geqslant 1} 2^{x-s} \Gamma\left(\frac{r}{k}\right) Q^{-1}\left(\frac{r}{k}, 2^{x-s}\right) .
\end{aligned}
$$

By a similar argument, (5.24) also holds when $h \geqslant 1$. (When $r=k,(5.24)$ reduces to $\lfloor\alpha-\beta\rfloor+2^{\{\alpha-\beta\}}-1$, as in [12].)

We next compute $\sum_{v \text { good }} \mathbb{E}\left[\xi_{r, v} \mathbb{1}\left[\xi_{r, v} \leqslant h\right]\right]$. By definition, if $v$ is good, then $n_{v}=$ $2^{m-t}-1$ with $l / 2 \leqslant t \leqslant L$. Let $u_{r, t}(x)$ be the probability density function of $\xi_{r, v}$. Differentiating (5.11) shows that uniformly for all $t \leqslant L$ and $x \geqslant m^{-5}$,

$$
u_{r, t}(x)=\left(1+O\left(\frac{\log m}{m}\right)^{\frac{1}{k}}\right) \hat{u}_{r, t}(x)
$$


where

$$
\hat{u}_{r, t}(x)=\frac{n}{m^{2} n_{v}} \exp \left(Q^{-1}\left(\frac{r}{k}, \frac{n x}{\Gamma\left(\frac{r}{k}\right) m n_{v}}\right)\right) Q^{-1}\left(\frac{r}{k}, \frac{n x}{\Gamma\left(\frac{r}{k}\right) m n_{v}}\right)^{1-\frac{r}{k}} .
$$

Using again the derivative formula (4.11), one can verify that

$$
\frac{\partial}{\partial x} \hat{U}_{r, t}(x)=x \hat{u}_{r, t}(x)
$$

where

$$
\hat{U}_{r, t}(x) \stackrel{\text { def }}{=} \frac{n_{v}}{n} \Gamma\left(1+\frac{r}{k}, Q^{-1}\left(\frac{r}{k}, \frac{n x}{\Gamma\left(\frac{r}{k}\right) m n_{v}}\right)\right)-\frac{x}{m} Q^{-1}\left(\frac{r}{k}, \frac{n x}{\Gamma\left(\frac{r}{k}\right) m n_{v}}\right) .
$$

Note also that $\hat{u}_{r, t}(x)=0$ if $\frac{n x}{\Gamma(1 / k) m n_{v}} \geqslant 1$. Thus

$$
\begin{aligned}
\mathbb{E}\left[\xi_{r, v} \mathbb{1}\left[\xi_{r, v} \leqslant h\right]\right]=\int_{m^{-5}}^{h} x u_{t}(x) \mathrm{d} x+\mathbb{E}\left[\xi_{r, v} \mathbb{1}\left[\xi_{r, v} \leqslant m^{-5}\right]\right] \\
=\left(1+O\left(\frac{\log m}{m}\right)^{\frac{1}{k}}\right)\left(\hat{U}_{r, t}\left(\frac{\Gamma\left(\frac{r}{k}\right) m n_{v}}{n} \wedge h\right)-\hat{U}_{r, t}\left(m^{-5}\right)\right)+o\left(m^{-2}\right) \\
=\left(1+O\left(\frac{\log m}{m}\right)^{\frac{1}{k}}\right) \hat{U}_{r, t}\left(\frac{\Gamma\left(\frac{r}{k}\right) m n_{v}}{n} \wedge h\right)+o\left(m^{-2}\right),
\end{aligned}
$$

where we use $\hat{U}_{r, t}\left(m^{-5}\right)=o\left(m^{-2}\right)$, which follows from the inequalities (5.7), (5.8) and (5.9).

If $t \geqslant l+1$, then

$$
\frac{n h}{\Gamma\left(\frac{r}{k}\right) m n_{v}} \geqslant \frac{2^{\beta-\alpha} \Gamma\left(\frac{r}{k}\right)}{\Gamma\left(\frac{r}{k}\right)} \frac{2^{m+\alpha+o(1)}}{2^{l+\beta+o(1)} 2^{m-l-1}}=2^{1+o(1)}>1,
$$

for $n$ large. Thus (5.30) reduces to

$$
\begin{aligned}
\mathbb{E}\left[\xi_{r, v} \mathbb{1}\left[\xi_{r, v} \leqslant h\right]\right] & =\left(1+O\left(\frac{\log m}{m}\right)^{\frac{1}{k}}\right) \hat{U}_{r, t}\left(\frac{\Gamma\left(\frac{r}{k}\right) m n_{v}}{n}\right)+o\left(m^{-2}\right) \\
& =(1+o(1)) \frac{n_{v}}{n} \Gamma\left(1+\frac{r}{k}\right)+o\left(m^{-2}\right) .
\end{aligned}
$$

If $t \leqslant l$, then

$$
\frac{n h}{\Gamma\left(\frac{r}{k}\right) m n_{v}} \leqslant \frac{2^{\beta-\alpha} \Gamma\left(\frac{r}{k}\right)}{\Gamma\left(\frac{r}{k}\right)} \frac{2^{m+\alpha+o(1)}}{2^{l+\beta+o(1)} 2^{m-l}}=1^{1+o(1)},
$$

and (5.30) reduces to

$$
\mathbb{E}\left[\xi_{r, v} \mathbb{1}\left[\xi_{r, v} \leqslant h\right]\right]=\left(1+O\left(\frac{\log m}{m}\right)^{\frac{1}{k}}\right) \hat{U}_{r, t}(h)+o\left(m^{-2}\right) .
$$


So we distinguish three cases, $l / 2 \leqslant t \leqslant l, l<t<L$, and $t=L$, which we refer to as the low part, the high part, and the last part.

The number of good nodes $v$ with $n_{v}=2^{m-t}-1$, is given by (5.12). Thus for the low part, i.e., when $v$ is a good node with $n_{v}=2^{m-t}$ and $l / 2 \leqslant t \leqslant l$,

$$
\begin{aligned}
\mu_{1} \stackrel{\text { def }}{=} & \sum_{v \text { good and low }} \mathbb{E}\left[\xi_{r, v} \mathbb{1}\left[\xi_{r, v} \leqslant h\right]\right] \\
= & \sum_{l / 2 \leqslant t \leqslant l}\left(2^{t+\alpha_{n}}+O(1)\right)\left(\left(1+O\left(\frac{\log m}{m}\right)^{\frac{1}{k}}\right) \hat{U}_{r, t}(h)+o\left(m^{-2}\right)\right) \\
= & \sum_{\mathrm{s}=1}^{\infty} \exp \left(-Q^{-1}\left(\frac{r}{k}, 2^{-\mathrm{s}}\right)\right) Q^{-1}\left(\frac{r}{k}, 2^{-\mathrm{s}}\right)^{\frac{r}{k}} \\
& \quad-\sum_{\mathrm{s}=1}^{\infty} 2^{-\mathrm{s}} \Gamma\left(\frac{r}{k}\right) Q^{-1}\left(\frac{r}{k}, 2^{-\mathrm{s}}\right)+2 \Gamma\left(1+\frac{r}{k}\right)+o(1),
\end{aligned}
$$

where the result has been simplified using (5.23). (The convergence of this sum follows from (5.8) and (5.9).) For the high part, i.e., when $v$ is a good node with $n_{v}=2^{m-t}$ and $l<t<L$,

$$
\begin{aligned}
\mu_{2} & \stackrel{\text { def }}{=} \sum_{v \text { good and high }} \mathbb{E}\left[\xi_{r, v} \mathbb{1}\left[\xi_{r, v} \leqslant h\right]\right] \\
& =\sum_{l<t<L}\left(2^{t+\alpha_{n}}+O(1)\right)\left((1+o(1)) \frac{n_{v}}{n} \Gamma\left(1+\frac{r}{k}\right)+o\left(m^{-2}\right)\right) \\
& =\Gamma\left(1+\frac{r}{k}\right)(L-l-1)+o(1) .
\end{aligned}
$$

And for the last part, i.e., when $v$ is good node with $n_{v}=2^{m-L}$,

$$
\begin{aligned}
\mu_{3} & \stackrel{\text { def }}{=} \sum_{v \text { good and last }} \mathbb{E}\left[\xi_{r, v} \mathbb{1}\left[\xi_{r, v} \leqslant h\right]\right] \\
& =\left(\left(2-2^{\alpha_{n}}\right) 2^{L}+O(1)\right)\left((1+o(1)) \frac{n_{v}}{n} \Gamma\left(1+\frac{r}{k}\right)+o\left(m^{-2}\right)\right) \\
& =2^{-\alpha}\left(2-2^{\alpha}\right) \Gamma\left(1+\frac{r}{k}\right)+o(1) .
\end{aligned}
$$

Together with (5.24),

$$
\begin{aligned}
& \sum_{v \text { good }} \mathbb{E}\left[\xi_{r, v} \mathbb{1}\left[\xi_{r, v} \leqslant h\right]\right] \\
& =\mu_{1}+\mu_{2}+\mu_{3}+o(1) \\
& \rightarrow f_{r, k, \gamma}+\Gamma\left(1+\frac{r}{k}\right)\left(2^{1-\alpha}+\alpha-\beta-\ell+L\right)-\int_{h}^{1} x \mathrm{~d} \nu_{r, k, \gamma}(x),
\end{aligned}
$$


where

$$
\begin{aligned}
f_{r, k, \gamma} \stackrel{\text { def }}{=} & \sum_{t=1}^{\infty} \exp \left(-Q^{-1}\left(\frac{r}{k}, 2^{\left\{\gamma-\lg \Gamma\left(\frac{r}{k}\right)\right\}-t}\right)\right) Q^{-1}\left(\frac{r}{k}, 2^{\left\{\gamma-\lg \Gamma\left(\frac{r}{k}\right)\right\}-t}\right)^{\frac{r}{k}} \\
& +\sum_{t=1}^{\infty}-2^{\left\{\gamma-\lg \Gamma\left(\frac{r}{k}\right)\right\}-t} \Gamma\left(\frac{r}{k}\right) Q^{-1}\left(\frac{r}{k}, 2^{\left\{\gamma-\lg \Gamma\left(\frac{r}{k}\right)\right\}-t}\right) \\
& +\Gamma\left(1+\frac{r}{k}\right)\left(2^{\left\{\gamma-\lg \Gamma\left(\frac{r}{k}\right)\right\}}-\left\{\gamma-\lg \Gamma\left(\frac{r}{k}\right)\right\}-\lg \Gamma\left(\frac{r}{k}\right)-1\right) .
\end{aligned}
$$

(The fact that $f_{r, k, \gamma}<\infty$ follows from the inequalities (5.7), (5.8), (5.9).) When $k=r$, the above is simply $2^{\gamma}-\gamma-1$, as in Theorem 1.1 of [12].

\subsection{Proof of Lemma 5.1 (iv)}

By the upper bound of $\mathrm{d} \nu_{r, k, \gamma} / \mathrm{d} x=\sum_{s \geqslant 1} g_{r}(s, x)$ in (5.17), $\int_{0}^{h} x^{2} \mathrm{~d} \nu_{r, k, \gamma}(x)<\infty$. Thus we are allowed to write this integral as

$$
\int_{0}^{h} x^{2} \mathrm{~d} \nu_{r, k, \gamma}(x)=\sum_{i=-1}^{-\infty} \int_{h 2^{i}}^{h 2^{i+1}} x^{2} \mathrm{~d} \nu_{r, k, \gamma}(x)=\sum_{s \geqslant 1} \sum_{i=-1}^{-\infty} \int_{h 2^{i}}^{h 2^{i+1}} x^{2} g_{r}(s, x) \mathrm{d} x .
$$

Recall that for $x \in\left(2^{i} h, 2^{i+1} h\right), g_{r}(s, x)=\hat{g}_{r}(s, x, i)$ (see (5.19)). Using the derivative formula (4.11), one can verify that

$$
\frac{\partial}{\partial x} \widetilde{G}_{r}(s, x, i)=x^{2} \hat{g}_{r}(s, x, i)
$$

where

$$
\begin{aligned}
\widetilde{G}_{r}(s, x, i) \stackrel{\text { def }}{=} & 2 x \exp \left(-Q^{-1}\left(\frac{r}{k}, \frac{2^{-i-t+\alpha-\beta} x}{\Gamma\left(\frac{r}{k}\right)}\right)\right) Q^{-1}\left(\frac{r}{k}, \frac{2^{-i-t+\alpha-\beta} x}{\Gamma\left(\frac{r}{k}\right)}\right)^{r / k} \\
& -x^{2} 2^{\alpha-\beta-i-t} Q^{-1}\left(\frac{r}{k}, \frac{2^{-i-t+\alpha-\beta} x}{\Gamma\left(\frac{r}{k}\right)}\right) \\
& +\frac{2 r x}{k} \Gamma\left(\frac{r}{k}, Q^{-1}\left(\frac{r}{k}, \frac{2^{-i-t+\alpha-\beta} x}{\Gamma\left(\frac{r}{k}\right)}\right)\right) \\
& -2^{-\alpha+\beta+i+\frac{k-2 r}{k}+t} \Gamma\left(\frac{2 r}{k}, 2 Q^{-1}\left(\frac{r}{k}, \frac{2^{-i-t+\alpha-\beta} x}{\Gamma\left(\frac{r}{k}\right)}\right)\right) \\
& -\frac{r 2^{-\alpha+\beta+i+t}}{k} \Gamma\left(\frac{r}{k}, Q^{-1}\left(\frac{r}{k}, \frac{2^{-i-t+\alpha-\beta} x}{\Gamma\left(\frac{r}{k}\right)}\right)\right)^{2} .
\end{aligned}
$$

Thus

$$
\int_{h 2^{i}}^{h 2^{i+1}} x^{2} g_{r}(s, x) \mathrm{d} x=\int_{h 2^{i}}^{h 2^{i+1}} x^{2} \hat{g}_{r}(s, x, i) \mathrm{d} x=\widetilde{G}_{r}\left(s, h 2^{i+1}, i\right)-\widetilde{G}_{r}\left(s, h 2^{i}, i\right) .
$$


Summing (5.43) over $i$ and $s$ as in (5.40)

$$
\begin{aligned}
\int_{0}^{h} x^{2} \mathrm{~d} \nu_{r, k, \gamma}(x)=2^{\beta-\alpha} & \left(\frac{3 r \Gamma\left(\frac{r}{k}\right)^{2}}{k}-4^{1-\frac{r}{k}} \Gamma\left(\frac{2 r}{k}\right)-\sum_{t=1}^{\infty} 2^{-t} \Gamma\left(\frac{r}{k}\right)^{2} Q^{-1}\left(\frac{r}{k}, 2^{-t}\right)\right. \\
& +2 \sum_{t=1}^{\infty} \exp \left(-Q^{-1}\left(\frac{r}{k}, 2^{-t}\right)\right) \Gamma\left(\frac{r}{k}\right) Q^{-1}\left(\frac{r}{k}, 2^{-t}\right)^{r / k} \\
& \left.-2^{1-\frac{2 r}{k}} \sum_{t=1}^{\infty} 2^{t} \Gamma\left(\frac{2 r}{k}, 2 Q^{-1}\left(\frac{r}{k}, 2^{-t}\right)\right)\right) .
\end{aligned}
$$

(The convergence of this sum follows from (5.8) and (5.9).) Note that this is simply $3 \cdot 2^{\beta-\alpha-1}$ for $k=r$, as in Lemma 2.5 of [12].

We next compute $\sum_{v \text { good }} \operatorname{Var}\left(\xi_{r, v} \mathbb{1}\left[\xi_{r, v} \leqslant h\right]\right)$. Using the estimation (5.32) and (5.34), we see that

$$
\sum_{v \text { good }} \mathbb{E}\left[\xi_{r, v} \mathbb{1}\left[\xi_{r, v} \leqslant h\right]\right]^{2}=o(1) .
$$

Thus it suffices to compute $\sum_{v \text { good }} \mathbb{E}\left[\xi_{r, v}^{2} \mathbb{1}\left[\xi_{r, v} \leqslant h\right]\right]$.

Let $v$ be a good node with $n_{v}=2^{m-t}$ and $l / 2 \leqslant t \leqslant l$. Then using (5.26),

$$
\begin{aligned}
\mathbb{E}\left[\xi_{r, v}^{2} \mathbb{1}\left[\xi_{r, v} \leqslant h\right]\right] & =\int_{m^{-5}}^{h} x^{2} u_{t}(x) \mathrm{d} x+o\left(m^{-2}\right) \\
& =\left(1+O\left(\frac{\log m}{m}\right)^{\frac{1}{k}}\right) \int_{m^{-5}}^{h} x^{2} \hat{u}_{r, t}(x) \mathrm{d} x+o\left(m^{-2}\right) .
\end{aligned}
$$

Using again the derivative formula (4.11), one can verify that

$$
\frac{\partial}{\partial x} \widetilde{U}_{r, t}(x)=x^{2} \hat{u}_{r, t}(x)
$$

where

$$
\begin{aligned}
\widetilde{U}_{r, t}(x) \stackrel{\text { def }}{=} & \frac{2 x n_{v}}{n} \exp \left(-Q^{-1}\left(\frac{r}{k}, \frac{n x}{m \Gamma\left(\frac{r}{k}\right) n_{v}}\right)\right) Q^{-1}\left(\frac{r}{k}, \frac{n x}{m \Gamma\left(\frac{r}{k}\right) n_{v}}\right)^{r / k} \\
& -\frac{m r n_{v}^{2}}{k n^{2}} \Gamma\left(\frac{r}{k}, Q^{-1}\left(\frac{r}{k}, \frac{n x}{m \Gamma\left(\frac{r}{k}\right) n_{v}}\right)\right)^{2}-\frac{x^{2}}{m} Q^{-1}\left(\frac{r}{k}, \frac{n x}{m \Gamma\left(\frac{r}{k}\right) n_{v}}\right) \\
& -\frac{m 2^{\frac{k-2 r}{k}} n_{v}^{2}}{n^{2}} \Gamma\left(\frac{2 r}{k}, 2 Q^{-1}\left(\frac{r}{k}, \frac{n x}{m \Gamma\left(\frac{r}{k}\right) n_{v}}\right)\right) \\
& +\frac{2 r x n_{v}}{k n} \Gamma\left(\frac{r}{k}, Q^{-1}\left(\frac{r}{k}, \frac{n x}{m \Gamma\left(\frac{r}{k}\right) n_{v}}\right)\right) .
\end{aligned}
$$


Recall that $\hat{u}_{r, t}(x)=0$ if $\frac{n x}{\Gamma(1 / k) m n_{v}} \geqslant 1$. Thus

$$
\begin{aligned}
& \mathbb{E}\left[\xi_{r, v}^{2} \mathbb{\mathbb { 1 }}\left[\xi_{r, v} \leqslant h\right]\right]=\int_{m^{-5}}^{h} x^{2} u_{t}(x) \mathrm{d} x+\mathbb{E}\left[\xi_{r, v} \mathbb{1}\left[\xi_{r, v} \leqslant m^{-5}\right]\right] \\
& \quad=\left(1+O\left(\frac{\log m}{m}\right)^{\frac{1}{k}}\right)\left(\widetilde{U}_{r, t}\left(\frac{\Gamma\left(\frac{r}{k}\right) m n_{v}}{n} \wedge h\right)-\widetilde{U}_{r, t}\left(m^{-5}\right)\right)+o\left(m^{-2}\right) \\
& =\left(1+O\left(\frac{\log m}{m}\right)^{\frac{1}{k}}\right) \widetilde{U}_{r, t}\left(\frac{\Gamma\left(\frac{r}{k}\right) m n_{v}}{n} \wedge h\right)+o\left(m^{-2}\right),
\end{aligned}
$$

where we use $\widetilde{U}_{r, t}\left(m^{-5}\right)=o\left(m^{-2}\right)$, which follows from the inequalities (5.7), (5.8) and (5.9).

The number of good nodes $v$ with $n_{v}=2^{m-t}-1$, is given by (5.12). We again separate good nodes into the low part $(l / 2 \leqslant t \leqslant l)$, the high part $(l<t<L)$ and the last part $(t=L)$ as in subsection 5.3. For the low part, i.e., when $v$ is a good node with $n_{v}=2^{m-t}$ and $l / 2 \leqslant t \leqslant l$,

$$
\begin{aligned}
\sigma_{1} \stackrel{\text { def }}{=} & \sum_{v \text { good and low }} \mathbb{E}\left[\xi_{r, v}^{2} \mathbb{1}\left[\xi_{r, v} \leqslant h\right]\right] \\
= & \sum_{l / 2 \leqslant t \leqslant l}\left(2^{t+\alpha_{n}}+O(1)\right)\left(1+O\left(\frac{\log m}{m}\right)^{\frac{1}{k}}\right)\left(\widetilde{U}_{r, t}(h)+o\left(m^{-2}\right)\right) \\
= & 2^{\beta-\alpha}\left(\sum_{\mathrm{s}=1}^{\infty} 2 \exp \left(-Q^{-1}\left(\frac{r}{k}, 2^{-\mathrm{s}}\right)\right) \Gamma\left(\frac{r}{k}\right) Q^{-1}\left(\frac{r}{k}, 2^{-\mathrm{s}}\right)^{r / k}\right. \\
& \frac{2 r \Gamma\left(\frac{r}{k}\right)^{2}}{k}-2^{1-\frac{2 r}{k}} \Gamma\left(\frac{2 r}{k}\right)-\sum_{\mathrm{s}=1}^{\infty} 2^{-\mathrm{s}} \Gamma\left(\frac{r}{k}\right)^{2} Q^{-1}\left(\frac{r}{k}, 2^{-\mathrm{s}}\right) \\
& \left.-\sum_{\mathrm{s}=1}^{\infty} 2^{-\frac{2 r}{k}+\mathrm{s}+1} \Gamma\left(\frac{2 r}{k}, 2 Q^{-1}\left(\frac{r}{k}, 2^{-\mathrm{s}}\right)\right)\right)
\end{aligned}
$$

For the high part, i.e., when $v$ is a good node with $n_{v}=2^{m-t}$ and $l<t<L$,

$$
\begin{aligned}
\sigma_{2} & \stackrel{\text { def }}{=} \sum_{v \text { good and high }} \mathbb{E}\left[\xi_{r, v}^{2} \mathbb{1}\left[\xi_{r, v} \leqslant h\right]\right] \\
& =\sum_{l<t<L}\left(2^{t+\alpha_{n}}+O(1)\right)\left(1+O\left(\frac{\log m}{m}\right)^{\frac{1}{k}}\right)\left(\widetilde{U}_{r, t}\left(\frac{\Gamma\left(\frac{r}{k}\right) m n_{v}}{n}\right)+o\left(m^{-2}\right)\right) \\
& =2^{\beta-\alpha}\left(\frac{r \Gamma\left(\frac{r}{k}\right)^{2}}{k}-2^{1-\frac{2 r}{k}} \Gamma\left(\frac{2 r}{k}\right)\right)+o(1) .
\end{aligned}
$$


And for the last part, i.e., when $v$ is good node with $n_{v}=2^{m-L}$,

$$
\begin{aligned}
\sigma_{3} & \stackrel{\text { def }}{=} \sum_{v \text { good and last }} \mathbb{E}\left[\xi_{r, v}^{2} \mathbb{1}\left[\xi_{r, v} \leqslant h\right]\right] \\
& =\left(\left(2-2^{\alpha_{n}}\right) 2^{L}+O(1)\right)\left(1+O\left(\frac{\log m}{m}\right)^{\frac{1}{k}}\right)\left(\widetilde{U}_{r, t}\left(\frac{\Gamma\left(\frac{r}{k}\right) m n_{v}}{n}\right)+o\left(m^{-2}\right)\right) \\
& =o(1) .
\end{aligned}
$$

Therefore,

$$
\sum_{v: h(v) \leqslant L} \operatorname{Var}\left(\xi_{r, v} \mathbb{1}\left[\xi_{r, v} \leqslant h\right]\right)=\sigma_{1}+\sigma_{2}+\sigma_{3}+o(1) \rightarrow \int_{0}^{h} x^{2} \mathrm{~d} \nu_{r, k, \gamma}(x),
$$

where the limit is given by (5.44). Thus we have completed the whole proof of Lemma 5.1.

\section{References}

[1] L. Addario-Berry, N. Broutin, and C. Holmgren. Cutting down trees with a Markov chainsaw. Ann. Appl. Probab., 24(6):2297-2339, 2014.

[2] X. S. Cai, L. Devroye, C. Holmgren, and F. Skerman. K-cuts on a Path. In Algorithms and Complexity, Lecture Notes in Computer Science, pages 112-123. Springer International Publishing.

[3] X. S. Cai, C. Holmgren, L. Devroye, and F. Skerman. K-cut on paths and some trees. Electron. J. Probab., 24, 2019.

[4] X. S. Cai and J. L. López. A note on the asymptotic expansion of the Lerch's transcendent. Integral Transforms and Special Functions, 30(10):844-855, Oct. 2019.

[5] D. Dagon, G. Gu, C. P. Lee, and W. Lee. A taxonomy of botnet structures. In Twenty-Third Annual Computer Security Applications Conference (ACSAC 2007), pages 325-339, 2007.

[6] NIST Digital Library of Mathematical Functions. Release 1.0.16 of 2017-09-18.

[7] M. Drmota, A. Iksanov, M. Moehle, and U. Roesler. A limiting distribution for the number of cuts needed to isolate the root of a random recursive tree. Random Structures \&f Algorithms, 34(3):319-336, 2009.

[8] R. Durrett. Probability: Theory and Examples, volume 31 of Cambridge Series in Statistical and Probabilistic Mathematics. Cambridge University Press, Cambridge, fourth edition, 2010.

[9] C. Holmgren. Random records and cuttings in binary search trees. Combin. Probab. Comput., 19(3):391-424, 2010.

[10] C. Holmgren. A weakly 1-stable distribution for the number of random records and cuttings in split trees. Adv. in Appl. Probab., 43(1):151-177, 2011. 
[11] A. Iksanov and M. Möhle. A probabilistic proof of a weak limit law for the number of cuts needed to isolate the root of a random recursive tree. Electron. Comm. Probab., 12:28-35, 2007.

[12] S. Janson. Random records and cuttings in complete binary trees. In Mathematics and Computer Science III, pages 241-253, Basel, 2004. Birkhäuser Basel.

[13] S. Janson. Random cutting and records in deterministic and random trees. Random Structures \&5 Algorithms, 29(2):139-179, 2006.

[14] S. Janson. Probability asymptotics: Notes on notation. ArXiv e-prints, Aug. 2011.

[15] O. Kallenberg. Foundations of Modern Probability. Probability and Its Applications (New York). Springer-Verlag, New York, second edition, 2002.

[16] A. Meir and J. Moon. Cutting down recursive trees. Mathematical Biosciences, 21(3):173 - 181, 1974.

[17] A. Meir and J. W. Moon. Cutting down random trees. J. Austral. Math. Soc., 11:313-324, 1970. 